\title{
Finitely coordinated models for low-temperature phases of amorphous systems
}

\author{
Reimer Kühn ${ }^{1}$, Jort van Mourik ${ }^{2}$, Martin Weigt ${ }^{3}$ and Annette Zippelius ${ }^{4}$ \\ ${ }^{1}$ Department of Mathematics, King's College London, Strand, London WC2R 2LS, UK \\ ${ }^{2}$ NCRG, Aston University, Aston Triangle, Birmingham B4 7ET, UK \\ ${ }^{3}$ Institute for Scientific Interchange, Viale S. Severo 65, 10133 Torino, Italy \\ ${ }^{4}$ Institut für Theoretische Physik, Universität Göttingen \\ Friedrich-Hund-Platz 1 , 37077 Göttingen, Germany
}

November 21, 2018

\begin{abstract}
We introduce models of heterogeneous systems with finite connectivity defined on random graphs to capture finite-coordination effects on the low-temperature behavior of finite dimensional systems. Our models use a description in terms of small deviations of particle coordinates from a set of reference positions, particularly appropriate for the description of low-temperature phenomena. A Born-von-Karman type expansion with random coefficients is used to model effects of frozen heterogeneities. The key quantity appearing in the theoretical description is a full distribution of effective single-site potentials which needs to be determined self-consistently. If microscopic interactions are harmonic, the effective single-site potentials turn out to be harmonic as well, and the distribution of these single-site potentials is equivalent to a distribution of localization lengths used earlier in the description of chemical gels. For structural glasses characterized by frustration and anharmonicities in the microscopic interactions, the distribution of single-site potentials involves anharmonicities of all orders, and both single-well and double well potentials are observed, the latter with a broad spectrum of barrier heights. The appearance of glassy phases at low temperatures is marked by the appearance of asymmetries in the distribution of single-site potentials, as previously observed for fully connected systems. Double-well potentials with a broad spectrum of barrier heights and asymmetries would give rise to the well known universal glassy low temperature anomalies when quantum effects are taken into account.
\end{abstract}




\section{Introduction}

Understanding the behaviour of amorphous systems, both at elevated and and at low temperatures, continues to be a challenging problem.

Structural glasses, for instance, show intriguing dynamic and thermodynamic behaviour when they are formed, e.g., by cooling from the liquid state [1]. A very sharp rise of relaxation times from microscopic to macroscopically large within a fairly narrow temperature interval entails that such systems will fall out of (local) equilibrium at a (cooling-rate dependent) calorimetric glass-transition temperature $T_{g}$, accompanied by a sharp drop of the specific heat $c_{p}(T)$ and thermal expansion coefficient $\alpha(T)$. Signatures of a dynamic singularity are, however, observed in many glasses at a temperature $T_{c}$ larger than that of the calorimetric transition $[1,2,3]$. Such a dynamic singularity was shown to emerge within a mode coupling theory (MCT) - a description of the dynamics of supercooled liquids in terms of correlation functions - via non-linear feedback mechanisms and memory-effects. The dynamic singularity occurs at a temperature where the viscosity is still fairly low; for a detailed review, see [2]. Conversely, if the behaviour of the entropy of the supercooled liquid is extrapolated to lower temperatures, it would become smaller than the entropy of the crystal at the Kauzmann temperature $T_{K}$, which is speculated to mark the location of a hypothetical true equilibrium glass transition, as it would be observed in the limit of infinitely slow cooling [4]. The Kauzmann temperature is usually quite close to the temperature $T_{0}$ marking a hypothetical divergence of the viscosity when fitted according to a Vogel-FulcherTammann form, and the two temperatures are often identified (and ought to be according to classical semi-microscopic [5] and phenomenological [6] approaches to the glass transition).

In a series of seminal papers, Kirkpatrick, Thirumalai, and Wolynes [7, 8, 9] pointed out a connection between the transition to frozen order in certain spin-glass models and the structural glass transition. The spin-glass models in question exhibit an equilibrium phase transition to a low-temperature phase with one 1 step replica-symmetry broken structure of the matrix of spinglass oder parameters. The transition is discontinuous in the order parameters, but exhibits no latent heat. Remarkably there is also a dynamical freezing transition at a temperature $T_{d}$ that is different (and higher) than the temperature $T_{0}$ at which the equilibrium transition occurs $[7,8,9]$. The phenomenology is strikingly similar to that found in structural glasses, where the dynamical transition is associated with the mode coupling transition, while the equilibrium transition would be associated with a hypothetical equilibrium transition at the Kauzmann temperature $T_{k}$, and in both cases, the equilibrium transition appears to be driven by an entropy crisis. The analogy was further elucidated and strengthened by studies of spherical version of the $p$-spin interaction spin-glass model $[10,11]$, for which macroscopic dynamical evolution equations for correlation and response functions can be formulated in closed form and have in fact the structure of idealized MCT equations [11]. In this case - unlike in structural glasses - the off-equilibrium dynamics in the non-ergodic low-temperature phase is also understood in rather great detail $[12]$. 
There is, of course, a sense in which the analogy between the physics of spin-glasses in the $p$-spin class and the physics of structural glasses is purely formal, as spin-glasses are systems with quenched disorder describing the interaction of degrees of freedom that are localized at all temperatures, whereas in structural glasses the disorder is self-induced and localization occurs only in the low-temperature phase. The issue of quenched disorder can be addressed at least schematically by noting that models with quenched disorder can, and have been used as bonafide approximants to describe the physics of models with self-induced disorder [13, 14, 15], yet the issue of localization remains. Significant progress has, however, been made towards a first principles description of glassy thermodynamics by Mézard and Parisi [16], based on an investigation of weakly coupled real replicas of liquid systems, elaborating on and extending related ideas of Monasson [17], Franz and Parisi [18, 19]. A more recent development are socalled lattice glasses $[20,21,22,23,24,25]$, i.e. lattice gas models with discrete degrees of freedom on (generalized) Bethe lattices. These models are neither locally disordered nor of infinite connectivity, allowing in this way for an ordered, crystalline state, and showing again glassy behavior due to self-induced disorder.

On a quite different note, structural glasses and other amorphous systems were found to exhibit thermodynamic, dynamic and transport properties at low temperatures, which are strikingly different from those of their crystalline counterparts [26]. At temperatures below $1 \mathrm{~K}$, the specific heat of glasses varies approximately linearly with temperature $T$, the behaviour of the thermal conductivity is close to quadratic in $T$, whereas in crystals both quantities are cubic in temperature. The behaviour of acoustic and dielectric response is also anomalous in this temperature range, when compared with the corresponding behaviour of crystals [27].

At temperatures at around $10 \mathrm{~K}$ there is an anomaly of a different kind in the specific heat which appears as a bump in the ratio $C(T) / T^{3}$ — the so-called Bose peak. The thermal conductivity exhibits a plateau in the Bose peak region, and begins to rise again as the temperature is further increased.

Below $1 \mathrm{~K}$, the low-temperature anomalies, as the phenomena just described have become to be referred to, are attributed to the existence of tunnelling systems which are absent in ideal crystals [28, 29]. Tunnelling systems are thought to be realized as double-well configurations in the potential energy landscape, between which atoms, or groups of atoms can tunnel, even when thermal energies to overcome the intervening potential energy barriers are not available. Double-well potentials with a broad range of asymmetries and barrier heights can give rise to the broad spectrum of low-energy tunneling-excitations responsible for the anomalous thermal properties, whereas their resonant and relaxational interaction with the phonon bath creates anomalous response properties. At temperatures (energies) in the Bose peak region, the main excitations appear to be soft quasi-harmonic. Their origin and precise nature is much debated (e.g. [30, 31, 32, 33, 34]); in any case, strong hybridization between these states and the phonon bath is likely to be an important feature. 
One of the remarkable aspects of glassy low-temperature anomalies is their considerable degree of universality. Two main contending theorys are available to explain this fact: Following ideas of $\mathrm{Yu}$ and Leggett [35], universality has been understood as a collective effect due to interactions between quantized excitations, i.e. the tunnelling systems [36]; alternatively universality is regarded as a consequence of the irregularities of the potential energy landscape that are frozen in at the glass transition, leading to a broad spectrum of quantized low-energy tunneling excitations $[37,38]$. The potential energy landscape being itself a collective affair generated through the interactions of constituent particles, one is led to expect a certain degree of insensitivity to detail of the low-temperature anomalies. This line of reasoning has been made more explicit in [39], and the key feature responsible for the universality of the low-temperature anomalies was demonstrated to be a clear separation of the energy scales frozen in at the glass transition on the one hand side, and that responsible for the low temperature physics on the other hand side. A simple explanation for the mysterious so-called quantitative universality, according to which not only power-laws describing the variation of some thermodynamic functions with temperature, but also prefactors in these power-laws are insensitive to detail, was also provided [39].

On the other hand, the description of the glassy freezing transition in these models is not as one would want to have it for structural glasses, as the transition to the non-ergodic low-temperature phase is not of the discontinuous 1-step replica-symmetry breaking (RSB) type as in the $p$-spin models, so the description chosen in [37, 38, 39] does appear to miss essential aspects of glass transition physics. To be fair though, it should be mentioned that those models were initially constructed mainly to provide a description of low-temperature phenomena. However, being microscopic the question as to their high-temperature properties naturally arises, and in this regime one might regard them as inadequate in their present form. Conversely, regarding lowtemperature phenomena, it is difficult to conceive how models of the $p$-spin type would describe the physics of tunnelling systems; in their spherical mean-field variant in particular [10, 11], they would give rise to harmonic effective single-site potentials which could not support tunnelling systems. It is thus perhaps fair to say that a description of both, the low-temperature phenomena, and glass-transition physics within a single unified approach still eludes us.

The main purpose of the present investigation to improve upon the models investigated in $[37,38,39]$, and consider variants in which interactions are finitely coordinated, keeping them random though, as a schematic way of modelling a frozen glassy state. Our investigation will exploit the fact that that the understanding of dilute random systems - introduced many years ago by Viana and Bray [40] — has witnessed significant progress in the last few years, regarding their equilibrium properties [41, 42, 43], as well as their dynamic behaviour [44, 45, 46, 47, 48]. This progress was also the basis for the previously discussed lattice-glass models, which, however, due to their discrete nature do not allow for the description of low-temperature excitations.

It is hoped that the additional element of realism introduced by considering finitely coordinated systems, respecting in this feature an important property of finite dimensional systems, could help bring those models closer to reality. 
The remainder of the paper is organized as follows. In Sect. 2 we introduce our model, and demonstrate how a finitely coordinated random model arises as a natural candidate to describe the physics of a glassy low temperature phase. We will look in detail only at a simplified version with scalar degrees of freedom. We describe the solution of the model using replica and mean field techniques in Sect. 3. However, in Sect. 3.1, we evaluate only a replica-symmetric (RS) approximation to the full theory. It turns out to have a similar complexity as a 1-RSB solution in models with discrete degrees of freedom. The key quantity appearing in the theoretical description is a full distribution of effective single-site potentials which needs to be determined self-consistently. A population-based stochastic algorithm is used to solve these RS self-consistency equations. The general set-up is very flexible and can be investigated for a broad spectrums of assumptions about the nature of the microscopic interactions as well as the connectivity distributions.

In Sect. 4 we evaluate the theory for models in which the microscopic interactions are purely harmonic, in which case the effective single-site potentials turn out to be harmonic as well; the distribution of the single-site potentials is then equivalent to a distribution of localization lengths used earlier in the description of systems of such type $[49,50,51,52,53]$, which may be thought of as systems in their gel-phase. For structural glasses characterized by frustration and anharmonicities in the microscopic interactions, investigated in Sect 5, the distribution of single-site potentials involves anharmonicities of all orders, and both single-well and double well potentials are observed, the latter with a broad spectrum of barrier heights. Although initially designed as models for the description of the low-temperature phase, it makes perfect sense to consider them also at high temperatures, where the microscopic symmetries of the interaction energy are unbroken. The appearance of glassy phases, as the temperature is lowered is marked by the appearance of asymmetries in the distribution of single-site potentials, as previously observed for fully connected systems. We investigate the phase diagram for a model system of this kind and compute thermodynamic function across the transition. As in fully connected systems, the transition is found to be continuous in our anharmonic model, and in this aspect the model we are considering here remains deficient. We discuss possible remedies of this deficiency in a concluding section.

Details of some of the calculations were relegated to appendices. The paper closes with a summary and an outlook on future interesting lines of research.

\section{The Model}

We consider a many-particle system described by the Hamiltonian:

$$
H=\sum_{i} \frac{\boldsymbol{p}_{i}^{2}}{2 m}+U_{\mathrm{int}}\left(\left\{\boldsymbol{r}_{i}\right\}\right)
$$


with an interaction energy taking the form

$$
U_{\mathrm{int}}=\sum_{(i, j)} \phi\left(\boldsymbol{r}_{i}-\boldsymbol{r}_{j}\right)+\sum_{i} V\left(\boldsymbol{r}_{i}\right)
$$

Our initial goal is to attempt a microscopic, albeit schematic description of the physics of an amorphous random solid. We proceed as follows. To begin with, we decompose each particle coordinate $\boldsymbol{r}_{i}$ into a fixed reference coordinate $\boldsymbol{R}_{i}$ and a relative coordinate $\boldsymbol{u}_{i}$ characterizing the (small) excursion from their respective reference position. Supposing that the initial pair potential has an effectively finite range, the interaction potential can then be formulated in terms of local connectivity variables and a (random) potential depending only on the relative coordinates.

The connectivity is characterized by a matrix $\mathbf{C}$ with elements $c_{i j}$, such that $c_{i j}=1$ for close particles, for which $\left|\boldsymbol{R}_{i}-\boldsymbol{R}_{j}\right| \leq r_{c}$, with $r_{c}$ denoting the interaction range of the initial pair potential $\phi$, and $c_{i j}=0$ if $\left|\boldsymbol{r}_{i}-\boldsymbol{r}_{j}\right| \simeq\left|\boldsymbol{R}_{i}-\boldsymbol{R}_{j}\right| \gg r_{c}$ and so $\phi\left(\boldsymbol{r}_{i}-\boldsymbol{r}_{j}\right) \simeq 0$. The interaction potential (2) may then be rewritten as

$$
U_{\mathrm{int}}=\sum_{(i, j)} c_{i j} \phi_{i j}\left(\boldsymbol{u}_{i}-\boldsymbol{u}_{j}\right)+\sum_{i} V_{i}\left(\boldsymbol{u}_{i}\right)
$$

Here the symbol $(i, j)$ is used to denote pairs of sites; non-zero pair potentials $\phi_{i j}$ and single-site potentials $V_{i}$ may vary from pair to pair and site to site, thus introducing residual disorder (arising, e.g., from interactions between different species, random nonzero $\boldsymbol{R}_{i}-\boldsymbol{R}_{j}$, etc., in the case of pair potentials, and the coupling of different species to external fields in case of the single-site potentials); we will specify $\phi$ and $V$ only later. One may think of the $\phi_{i j}\left(\boldsymbol{u}_{i}-\boldsymbol{u}_{j}\right)$ as of a Born-von Karman expansion of the interaction potential about the reference positions, carried to all orders.

In case one is interested mainly in low-temperature phenomena, one would expect that some low order expansion of the $\phi_{i j}$ should be sufficient to capture the essential physics.

As a crucial last ingredient, which renders the model solvable, we consider connectivity matrices $\mathbf{C}=\left\{c_{i j}\right\}$ which are not created as a consequence of an underlying geometrical arrangement of the interacting particles as described above, but rather we take them to be random. We assume that the local coordination number $L_{i}$ of site $i$ is distributed according to a distribution $P_{C}(L)$, with finite average connectivity $C=\sum_{L} L P_{C}(L)=\frac{1}{N} \sum_{i} L_{i}$, and that the probability that two sites $i \neq j$ with coordination numbers $L_{i}, L_{j}$ are connected (i.e. that $c_{i j}=c_{j i}=1$ ) is proportional to $L_{i} L_{j}$ (no correlations). The adjacency matrices $\mathbf{C}$ in the ensemble determined by $P_{C}(L)$ then have the following distribution

$$
P_{c}\left(\mathbf{C} \mid\left\{L_{i}\right\}\right)=\frac{1}{\mathcal{N}} \prod_{(i, j)} p_{C}\left(c_{i j}\right) \delta_{c_{i j}, c_{j i}} \prod_{i} \delta_{L_{i}, \sum_{j(\neq i)} c_{i j}}
$$


where $\mathcal{N}$ is a normalization constant. It iturns out that the distribution (4) is independent of the conditioning $\left\{L_{i}\right\}$ in the large $N$ limit, for any typical realization of the set $\left\{L_{i}\right\}$ compatible with a given distribution

$$
P_{C}(L)=\frac{1}{N} \sum_{i} \delta_{L, L_{i}}
$$

of coordination numbers. A priori single bond probabilities

$$
p_{C}\left(c_{i j}\right)=\frac{C}{N} \delta_{c_{i j}, 1}+\left(1-\frac{C}{N}\right) \delta_{c_{i j}, 0} .
$$

compatible with the average connectivity $C$ are also included in (4), as a matter of convenience rather than necessity, as constraints coming from the full distribution of coordination numbers are also encoded in (4). Other, equivalent formulations are possible as noted in Appendix A. Note that for $P_{C}(L)=\frac{C^{L}}{L !} \exp (-C)$ the connectivity matrix is that of an Erdös-Renyi random graph.

The residual randomness in the pair and single-site potentials is described by normalized probability densities $P_{\phi}[\phi]$ and $P_{V}[V]$.

In its present formulation, the model can be solved within replica mean-field theory. The nature of the random pair and single-site potentials as well as the distribution of connectivities can be left open for the time being. Specifying them in different ways one has access to describing a variety of different physical systems.

\section{Replica Analysis}

The generator of all the relevant physical quantities is the free energy, $-N \beta f(\beta)=\ln \left(Z_{N}\right)$, and using the standard replica trick $\langle\log Z\rangle=\lim _{n \rightarrow 0} n^{-1} \log \left\langle Z^{n}\right\rangle$, we need to calculate the average of the $n$-fold replicated partition function over the bond-disorder, i.e. the $\mathbf{C}$, and the $\phi_{i j}$ :

$$
\left\langle Z_{N}^{n}\right\rangle_{\mathbf{C}, \phi}=\left\langle\int \prod_{i, a} d \boldsymbol{u}_{i}^{a} \exp \left\{-\left(\beta \sum_{(i, j), a} c_{i j} \phi_{i j}\left(\boldsymbol{u}_{i}^{a}-\boldsymbol{u}_{j}^{a}\right)+\sum_{i, a} V_{i}\left(\boldsymbol{u}_{i}^{a}\right)\right)\right\}\right\rangle_{\mathbf{C}, \phi}
$$

Free energies are manifestly self-averaging w.r.t. any form of (finite-dimensional) site disorder, which is why taking a $V$-average is not required here. It is useful to introduce the short-hand notation $\tilde{\boldsymbol{u}}=\left(\boldsymbol{u}^{1}, \ldots, \boldsymbol{u}^{n}\right)$ for a replicated vector, and the replica interaction (bond) and singlesite weights:

$$
\mathcal{U}_{b}(\tilde{\boldsymbol{u}}, \tilde{\boldsymbol{v}}, \phi) \equiv \exp \left(-\beta \sum_{a} \phi\left(\boldsymbol{u}^{a}-\boldsymbol{v}^{a}\right)\right), \quad \mathcal{U}_{s}(\tilde{\boldsymbol{u}}, V) \equiv \exp \left(-\beta \sum_{a} V\left(\boldsymbol{u}^{a}\right)\right) .
$$


The line of reasoning involved in performing the average over connectivity matrices for nonPoissonian connectivity distribution closely follows [54]. Constraints on the local connectivities in the $\mathbf{C}$-average are enforced via the identity

$$
\delta_{K_{i}, L_{i}}=\oint \frac{d z_{i}}{2 \pi \mathrm{i} z_{i}} z_{i}^{\left(K_{i}-L_{i}\right)}
$$

It is found that the average of the replicated partition function can be written as a functional integral over the 'replica density'

$$
\rho(\tilde{\boldsymbol{u}}) \equiv \frac{1}{N} \sum_{i} z_{i} \delta\left(\tilde{\boldsymbol{u}}-\tilde{\boldsymbol{u}}_{i}\right)
$$

Enforcing its definition in terms of conjugate variables $\hat{\rho}(\tilde{\boldsymbol{u}})$ we obtain after some standard steps (for details see appendix A):

$$
\left\langle Z_{N}^{n}\right\rangle_{\mathbf{C}, \phi}=\frac{1}{\mathcal{N}} \int \mathcal{D} \rho \mathcal{D} \hat{\rho} \exp \left\{N\left(\frac{C}{2}\left(G_{b}[\rho]-1\right)-G_{m}[\rho, \hat{\rho}]+G_{s}[\hat{\rho}]\right)\right\}
$$

where

$$
\begin{aligned}
G_{b}[\rho] & =\left\langle\int d \tilde{\boldsymbol{u}} d \tilde{\boldsymbol{v}} \rho(\tilde{\boldsymbol{u}}) \mathcal{U}_{b}(\tilde{\boldsymbol{u}}, \tilde{\boldsymbol{v}}, \phi) \rho(\tilde{\boldsymbol{v}})\right\rangle_{\phi} \\
G_{m}[\rho, \hat{\rho}] & =\int d \tilde{\boldsymbol{u}} d \tilde{\boldsymbol{v}} \hat{\rho}(\tilde{\boldsymbol{u}}) \rho(\tilde{\boldsymbol{u}}) \\
G_{s}[\hat{\rho}] & =\sum_{L} P_{C}(L)\left\langle\ln \int d \tilde{\boldsymbol{u}} \hat{\rho}^{L}(\tilde{\boldsymbol{u}}) \mathcal{U}_{s}(\tilde{\boldsymbol{u}}, V)\right\rangle_{V}
\end{aligned}
$$

The path integral is dominated by its saddle point, and functional variation with respect to $\rho(\tilde{\boldsymbol{u}})$, and $\hat{\rho}(\tilde{\boldsymbol{u}})$ leads to the stationarity conditions:

$$
\begin{aligned}
& \hat{\rho}(\tilde{\boldsymbol{u}})=C\left\langle\int d \tilde{\boldsymbol{v}} \mathcal{U}_{b}(\tilde{\boldsymbol{u}}, \tilde{\boldsymbol{v}}, \phi) \rho(\tilde{\boldsymbol{v}})\right\rangle_{\phi} \\
& \rho(\tilde{\boldsymbol{u}})=\sum_{L} P_{C}(L) L\left\langle\frac{\hat{\rho}^{L-1}(\tilde{\boldsymbol{u}}) \mathcal{U}_{s}(\tilde{\boldsymbol{u}}, V)}{\int d \tilde{\boldsymbol{v}} \hat{\rho}^{L}(\tilde{\boldsymbol{v}}) \mathcal{U}_{s}(\tilde{\boldsymbol{v}}, V)}\right\rangle_{V}
\end{aligned}
$$

\subsection{Replica Symmetry}

In order to be able to take the limit $n \rightarrow 0$ in (11)-(14) as required for the replica method, we need to make an ansatz for the replicated density $\rho$ in (10) and its conjugate $\hat{\rho}$, which assumes certain symmetry properties under permutation of the replicas. Within the present paper we 
shall only explicitly deal with a replica symmetric (RS) ansatz. Variants which would break the symmetry between replicas are fairly straightforward to write down (see Appendix A), but for the model class studied in the present paper they turn out to be extremely complex and difficult to handle numerically. The reader will appreciate this statement once the RS theory has been developed.

The RS ansatz describes a situation with unbroken replica symmetry, and without loss of generality both $\rho(\tilde{\boldsymbol{u}})$ and its conjugate $\hat{\rho}(\tilde{\boldsymbol{u}})$ can be written as (functional) superpositions of products of single replica functions of the form

$$
\begin{aligned}
& \rho(\tilde{\boldsymbol{u}})=\int \mathcal{D} \psi \pi[\psi] \prod_{a} \frac{\exp \left(-\beta \psi\left(\boldsymbol{u}^{a}\right)\right)}{Z[\psi]} \\
& \hat{\rho}(\tilde{\boldsymbol{u}})=C \int \mathcal{D} \hat{\psi} \hat{\pi}[\hat{\psi}] \prod_{a} \frac{\exp \left(-\beta \hat{\psi}\left(\boldsymbol{u}^{a}\right)\right)}{Z[\hat{\psi}]} .
\end{aligned}
$$

Here we use the convention

$$
Z[f] \equiv \int \mathrm{d} \boldsymbol{u} \exp (-\beta f(\boldsymbol{u}))
$$

i.e. the single replica functions in (15) and (16) are taken to have Gibbsian form, with functions $\psi$ and $\hat{\psi}$ denoting single-replica potentials that generate the Gibbs distributions in question. Note that $\mathcal{D} \psi$ and $\mathcal{D} \hat{\psi}$ are suitable measures in function space. With the prefactor $C$ as chosen in (16), it turns out that, both $\pi$ and $\hat{\pi}$ are normalized probability density functions (pdfs) over function space (see Appendix A). In terms of these specifications, the replicated partition function (11) may be re-expressed as a functional integral over $\pi$ and $\hat{\pi}$ in a way that allows to isolate the leading order in $n$ (i.e. $\mathcal{O}(n)$ in the limit $n \rightarrow 0$ ). One obtains an expression of the form

$$
\left\langle Z_{N}^{n}\right\rangle_{\mathbf{C}, \phi} \sim \int \mathcal{D} \pi \mathcal{D} \hat{\pi} \exp \left(n N\left\{\frac{C}{2} \mathcal{G}_{b}[\pi]-C \mathcal{G}_{m}[\pi, \hat{\pi}]+\sum_{L} P_{C}(L) \mathcal{G}_{s, L}[\hat{\pi}]\right\}\right)
$$

On introducing

$$
Z_{2}[f, g, h] \equiv \int \mathrm{d} \boldsymbol{u} \mathrm{d} \boldsymbol{v} \exp (-\beta(f(\boldsymbol{u})+g(\boldsymbol{v})+h(\boldsymbol{u}, \boldsymbol{v})))
$$

and short hands of the form $\mathcal{D} \pi[\psi]=\mathcal{D} \psi \pi[\psi]$ and $\{\mathcal{D} \hat{\pi}\}_{L} \equiv \prod_{\ell}^{L} \mathcal{D} \hat{\pi}\left[\hat{\psi}_{\ell}\right]$, one can express the three functionals appearing in (18) as

$$
\mathcal{G}_{b}[\pi] \simeq \int \mathcal{D} \pi\left[\psi_{1}\right] \mathcal{D} \pi\left[\psi_{2}\right]\left\langle\ln \left(\frac{Z_{2}\left[\psi_{1}, \psi_{2}, \phi\right]}{Z\left[\psi_{1}\right] Z\left[\psi_{2}\right]}\right)\right\rangle_{\phi}
$$




$$
\begin{aligned}
\mathcal{G}_{m}[\pi, \hat{\pi}] & \simeq \int \mathcal{D} \pi[\psi] \mathcal{D} \hat{\pi}[\hat{\psi}] \ln \left(\frac{Z[\psi+\hat{\psi}]}{Z[\psi] Z[\hat{\psi}]}\right), \\
\mathcal{G}_{s, L}[\hat{\pi}] & \simeq \int\{\mathcal{D} \hat{\pi}\}_{L}\left\langle\ln \left(\frac{Z\left[\sum_{\ell=1}^{L} \hat{\psi}_{\ell}+V\right]}{\prod_{\ell=1}^{L} Z\left[\hat{\psi}_{\ell}\right]}\right)\right\rangle_{V} .
\end{aligned}
$$

All $L$ (coordination number) summations are over non-negative integers (and the convention is used that an empty product evaluates to unity).

Note that one can easily identify a bond (i.e. pair-interaction) term $\mathcal{G}_{b}$, a mixture term $\mathcal{G}_{m}$, and coordination number $L$ dependent single site terms $\mathcal{G}_{s, L}$.

The precise form of the various functions and parameters is obtained from the stationarity condition with respect to variations of $\pi$, which involves solving stationarity conditions w.r.t. the conjugate functional and $\hat{\pi}$ as well (Lagrange multipliers $\lambda, \hat{\lambda}$ are introduced to take care of the normalization constraint on $\pi$ and $\hat{\pi}$ in the variational procedure). We finally obtain (for details see e.g. $[41,43])$ the following coupled set of integral equations for $\hat{\pi}$ and $\pi$ :

$$
\begin{aligned}
& \hat{\pi}[\hat{\psi}]=\int \mathcal{D} \pi[\psi]\langle\delta[\hat{\psi}-\hat{\Psi}[\psi, \phi]]\rangle_{\phi} \\
& \pi[\psi]=\sum_{L} \frac{L}{C} P_{C}(L) \int\{\mathcal{D} \hat{\pi}\}_{L-1}\left\langle\delta\left[\psi-\Psi\left[\left\{\hat{\psi}_{\ell}\right\}_{L-1}, V\right]\right]\right\rangle_{V},
\end{aligned}
$$

in which the functions $\Psi\left[\left\{\hat{\psi}_{\ell}\right\}_{L}, V\right]$ and $\hat{\Psi}[\psi, \phi]$ are defined as

$$
\begin{aligned}
\Psi\left[\left\{\hat{\psi}_{\ell}\right\}_{L}, V\right] & =\sum_{\ell}^{L} \hat{\psi}_{\ell}+V, \\
\hat{\Psi}[\psi, \phi] & =-\beta^{-1} \ln Z_{\psi, \phi},
\end{aligned}
$$

with

$$
Z_{\psi, \phi}(\boldsymbol{v}) \equiv \int d \boldsymbol{u} \exp (-\beta \psi(\boldsymbol{u})-\beta \phi(\boldsymbol{u}-\boldsymbol{v}))
$$

To summarize, we see that $\hat{\pi}[\hat{\psi}]$ can be obtained from sampling from $P_{\phi}[\phi]$ and $\pi[\psi]$, while $\pi[\psi]$ can be obtained from sampling from $\frac{L}{C} P_{C}(L), P_{V}[V]$, and $\hat{\pi}[\hat{\psi}]$. This provides a strategy of solving the fixed point equations via a population dynamics based algorithm.

In the saddle point the expression for the free energy (see Appendix B) simplifies to

$$
\begin{aligned}
\beta f(\beta)=\frac{C}{2} \int & \mathcal{D} \pi\left[\psi_{1}\right] \mathcal{D} \pi\left[\psi_{2}\right]\left\langle\ln Z_{2}\left[\psi_{1}, \psi_{2}, \phi\right]\right\rangle_{\phi} \\
& -\sum_{L} P_{C}(L) \int\{\mathcal{D} \hat{\pi}\}_{L}\left\langle\ln Z\left[\Psi\left[\{\hat{\psi}\}_{L}, V\right]\right]\right\rangle_{V} .
\end{aligned}
$$


The internal energy is given by:

$$
\begin{aligned}
E(\beta)=\frac{C}{2} \int \mathcal{D} \pi\left[\psi_{1}\right] \mathcal{D} \pi\left[\psi_{2}\right]\left\langle\langle\phi(\boldsymbol{u}-\boldsymbol{v})\rangle_{b, 2}\right\rangle_{\phi} \\
+\sum_{L} P_{C}(L) \int\{\mathcal{D} \hat{\pi}\}_{L}\left\langle\langle V(\boldsymbol{u})\rangle_{s, L}\right\rangle_{V},
\end{aligned}
$$

where $\langle\cdot\rangle_{b, 2}$ denotes the Gibbs average corresponding to the bond energy

$$
\hat{\Psi}_{b}\left[\psi_{1}, \psi_{2}, \phi\right](\boldsymbol{u}, \boldsymbol{v})=\psi_{1}(\boldsymbol{u})+\psi_{2}(\boldsymbol{v})+\phi(\boldsymbol{u}-\boldsymbol{v}),
$$

while $\langle\cdot\rangle_{s, L}$ is the Gibbs average corresponding to the single-site potential $\Psi\left[\left\{\hat{\psi}_{\ell}\right\}_{L}, V\right]$. Note that contributions to the internal energy arising from temperature-dependences of the distributions $\pi$ and $\hat{\pi}$ vanish owing to the stationarity condition on $f(\beta)$ w.r.t $\pi$ and $\hat{\pi}$.

Thermal averages of arbitrary single-site observables are evaluated as Gibbs averages in the ensemble (25) of effective single-site potentials $V_{\text {eff }}=\sum_{\ell}^{L} \hat{\psi}_{\ell}+V$, which are distributed according to

$$
P\left[V_{\text {eff }}\right]=\sum_{L} P_{C}(L) \int\{\mathcal{D} \hat{\pi}\}_{L}\left\langle\delta\left[V_{\text {eff }}-\Psi\left[\left\{\hat{\psi}_{\ell}\right\}_{L}, V\right]\right]\right\rangle_{V}
$$

i.e. for an arbitrary observable of the form $A=\frac{1}{N} \sum_{i} A\left(\boldsymbol{u}_{i}\right)$ we have that its thermal average is given by

$$
\begin{aligned}
\langle A\rangle & =\int \mathcal{D} P\left[V_{\mathrm{eff}}\right] \frac{\int \mathrm{d} \boldsymbol{u} A(\boldsymbol{u}) \exp \left(-\beta V_{\mathrm{eff}}(\boldsymbol{u})\right)}{Z\left[V_{\mathrm{eff}}\right]} \\
& =\sum_{L} P_{C}(L) \int\{\mathcal{D} \hat{\pi}\}_{L}\left\langle\frac{\int \mathrm{d} \boldsymbol{u} A(\boldsymbol{u}) \exp \left(-\beta \Psi\left[\{\hat{\psi}\}_{L}, V\right](\boldsymbol{u})\right)}{Z\left[\Psi\left[\{\hat{\psi}\}_{L}, V\right]\right]}\right\rangle_{V}
\end{aligned}
$$

Note that for Poissonian random graphs, the ensemble of single-replica potentials $\psi$ is equivalent to the ensemble of effective single-site potentials $V_{\text {eff }}$. This follows by comparison of (24) and (31) on noting that $\frac{L}{C} P_{C}(L)=P_{C}(L-1)$ for Poisson distributions. This implies in particular that the equivalence between the ensembles of single-replica potentials and effective single-site potentials is lost for systems with connectivity distributions other than Poissonian.

We note that an alternative way of obtaining the replica symmetric theory is via the Bethe-Peierls iterative method for obtaining free energies on (locally) tree-like structures [42]. Appendix C gives a sketch of the reasoning for the present model class.

This concludes the general framework. 


\subsection{Orthogonal Function Representation}

It should be clear that instead of working with the representation-free full functional set-up described above, we could have chosen to represent the single replica potentials $\psi$ and $\hat{\psi}$ in terms of their expansions using a suitable complete set of basis functions $\left\{\varphi_{\mu}\right\}$ :

$$
\psi(\boldsymbol{u})=\sum_{\mu} x_{\mu} \varphi_{\mu}(\boldsymbol{u}) \equiv \underline{x} \cdot \underline{\varphi}(\boldsymbol{u}),
$$

and analogously $\hat{\psi}(\boldsymbol{u})=\hat{x}_{\mu} \cdot \underline{\varphi}(\boldsymbol{u})$. Integrals over the function space are then replaced by multiple integrals over expansion coefficients. It is convenient to choose the basis functions $\left\{\varphi_{\mu}\right\}$ to be orthonormal with respect to a scalar product,

$$
\left(\varphi_{\mu} \mid \varphi_{\nu}\right)=\delta_{\mu, \nu}
$$

such that any function $f$ spanned by the $\left\{\varphi_{\mu}\right\}$ has an expansion

$$
f(\boldsymbol{u})=\underline{f} \cdot \underline{\varphi}(\boldsymbol{u}), \quad f_{\mu}=\left(f \mid \varphi_{\mu}\right) .
$$

Instead of (15) and (16) one would write

$$
\begin{aligned}
& \rho(\boldsymbol{u})=\int d \pi(\underline{x}) \prod_{a} \frac{\exp \left[-\beta \underline{x} \cdot \underline{\varphi}\left(\boldsymbol{u}^{a}\right)\right]}{Z(\underline{x})} \\
& \hat{\rho}(\boldsymbol{u})=C \int d \hat{\pi}(\underline{\hat{x}}) \prod_{a} \frac{\exp \left[-\beta \underline{\hat{x}} \cdot \underline{\varphi}\left(\boldsymbol{u}^{a}\right)\right]}{\hat{Z}(\underline{\hat{x}})}
\end{aligned}
$$

for the replica density and its conjugate, with $Z(\underline{x})$ and $\hat{Z}(\underline{\hat{x}})$ fixed by a normalization condition. Shorthands such as $d \pi(\underline{x}) \equiv d \underline{x} \pi(\underline{x})$ are being used in analogy to before.

Repeating the above line of reasoning one would obtain

$$
\begin{aligned}
\hat{\pi}(\underline{\hat{x}}) & =\int d \pi(\underline{x})\langle\delta(\underline{\hat{x}}-\underline{\hat{X}}(\underline{x}, \phi))\rangle_{\phi} \\
\pi(\underline{x}) & =\sum_{L} \frac{L}{C} P_{C}(L) \int \prod_{\ell}^{L-1} d \hat{\pi}\left(\underline{\hat{x}}_{\ell}\right)\left\langle\delta\left(\underline{x}-\underline{X}_{L-1}\left(\left\{\underline{\hat{x}}_{\ell}\right\}\right)\right)\right\rangle_{V}
\end{aligned}
$$

with

$$
\begin{aligned}
\underline{X}_{L-1}\left(\left\{\underline{\hat{x}}_{\ell}\right\}\right) & =(\underline{\varphi} \mid V)+\sum_{\ell}^{L-1} \underline{\hat{x}}_{\ell}, \\
\underline{\hat{X}}(\underline{x}, \phi) & =-\beta^{-1}\left(\underline{\varphi} \mid \ln Z_{\underline{x}, \phi}\right),
\end{aligned}
$$


and

$$
Z_{\underline{x}, \phi}(v)=\int d \boldsymbol{u} \exp (-\beta \underline{x} \cdot \underline{\varphi}(\boldsymbol{u})-\beta \phi(\boldsymbol{u}-\boldsymbol{v}))
$$

instead of (23)-(27). The distribution (31) of effective single site potentials translates in an obvious way to a corresponding distribution of their expansion coefficients in an orthogonal function representation, given by

$$
P\left(\underline{x}_{\text {eff }}\right)=\sum_{L} P_{C}(L) \int \prod_{\ell}^{L} d \hat{\pi}\left(\underline{\hat{x}}_{\ell}\right)\left\langle\delta\left(\underline{x}_{\mathrm{eff}}-\underline{X}_{L}\left(\left\{\underline{\hat{x}}_{\ell}\right\}\right)\right)\right\rangle_{V} .
$$

An obvious advantage of explicit representations of this form is that one may use it to formulate natural approximation schemes by truncating the expansions at some suitable finite order. A corresponding disadvantage would be that such truncations can be expected to be efficient only, if the system of basis functions is well adapted to the problem being studied.

\section{Harmonic Couplings}

The first model that we investigate is a random graph of harmonically coupled scalar degrees of freedom with a distribution of the coupling strengths. We assume that there is no single site potential, so that the system is translationally invariant. The interaction energy between connected vertices $(i, j)$ of the graph is given by

$$
\phi_{i j}\left(u_{i}-u_{j}\right)=\frac{k_{i j}}{2}\left(u_{i}-u_{j}\right)^{2}
$$

Models of this type have been used to describe gels; typically, uniform distributions of cross-link strengths $P\left(k_{i j}\right)=\delta\left(k_{i j}-k\right)$ where chosen in that context [49,50,51]. Alternatively models with random harmonic couplings on regular lattices [33] have been looked at in connection with Bose-peak phenomena. Note that [51], which — unlike our present modelling — provides a description preserving information about spatial structure, has recently been used to provide a microscopic underpinning of a phenomenological model of an elasticity theory for soft random solids based on randomly varying elastic constants [53]. In the present investigation, we combine elements of random structure with random values for existing couplings.

It turns out that the case of harmonically coupled degrees of freedom provides the only example where the population dynamics described above is closed within a parameter space of finite dimensionality. In all other cases, finite dimensional parameter spaces can only give variational approximations to a full solution.

It should be noted, however, that harmonically coupled systems have unique ground states and therefore miss essential elements of glassy low-temperature physics. E.g. they are unable to support two-level tunneling systems in their potential energy landscape. 
On the positive side, one would expect the replica symmetric approximation to be exact for such systems.

Since the local variables can take any real value, we take the modified Hermite polynomials $\varphi_{\mu}(u) \equiv \frac{1}{\sqrt{h_{\mu}}} H_{\mu}(u)$, with $h_{\mu}=\sqrt{\pi} 2^{\mu} \mu$ ! as the appropriate set of basis functions, in which the $H_{\mu}(u)$ are Hermite polynomials satisfying the recursion relation

$$
H_{\mu+1}(u)=2 u H_{\mu}(u)-2 \mu H_{\mu-1}(u), \quad \mu=0,1,2 . ., \infty, \quad H_{0}(u)=1 .
$$

The polynomials $\varphi_{\mu}$ are orthonormal with respect to the scalar product:

$$
(f \mid g) \equiv \int d u \exp \left(-u^{2}\right) f(u) g(u)
$$

In the present case the family of orthogonal polynomials can be truncated after $\varphi_{2}$, to leave the following set of basis functions:

$$
\left\{\varphi_{\mu}(u)\right\}=\left\{\frac{1}{\sqrt{h_{0}}}, \frac{2 u}{\sqrt{h_{1}}}, \frac{4 u^{2}-2}{\sqrt{h_{2}}}\right\}
$$

In the fixed point equations (39-41), the integral in (42), for $\phi(u-v)=\frac{k}{2}(u-v)^{2}$, is Gaussian and can be done analytically, such that the $\hat{X}_{\mu}$ are determined as:

$$
\hat{X}_{0}=x_{0}-\frac{1}{\beta} \ln \sqrt{\frac{2 \pi}{\beta k a}}-\frac{\sqrt{h_{0}}}{k a}\left(\frac{2 x_{1}^{2}}{h_{1}}+\frac{16 x_{2}^{2}}{h_{2}}\right), \quad \hat{X}_{1}=\frac{x_{1}}{a}, \quad \hat{X}_{2}=\frac{x_{2}}{a},
$$

where

$$
a \equiv 1+\frac{8 x_{2}}{\sqrt{h_{2}} k}
$$

thus leaving us with algebraic updates for the population dynamics only.

We observe that a positive $x_{2}$ generates a positive $\hat{X}_{2}$, thus guaranteeing stability starting from distributions $\pi(\underline{x})$ and $\hat{\pi}(\underline{\hat{x}})$ which are non-zero for $x_{2}, \hat{x}_{2} \in \mathbb{R}^{+}$only. We also note that the evolution of the $x_{0}$ and $\hat{x}_{0}$ distribution does not couple back into the distribution of nonconstant contribution to the $\psi(u)$ and $\hat{\psi}(u)$ respectively. Finally, it is interesting to observe that the distribution of $x_{1}, x_{2}, \hat{x}_{1}$ and $\hat{x}_{2}$, are temperature independent for the harmonic system, while the $x_{0}$ and $\hat{x}_{0}$ distributions exhibit a non-trivial temperature dependence.

The results are presented in the following figures. Fig 1 shows the distribution of the expansion coefficient $x_{2}$ of the single-replica potential in an expansion in modified Hermite polynomials as explained above, for a Poissonian random graph. As mentioned before, this is in the present case equivalent to the distribution of the expansion coefficient $x_{\mathrm{eff}, 2}$ of the (purely harmonic) effective 
single-site potential. The expansion coefficient $x_{\mathrm{eff}, 2}$ is in turn related to the localization length $\xi$ of the particles via

$$
\xi^{-2}=\beta \frac{8 x_{\mathrm{eff}, 2}}{\sqrt{h}_{2}} .
$$

The multiple peak structure in the distribution of localization lengths is notable, and related to the fact that localization lengths of different particles are to first approximation determined by their coordination - the clearly discernible 7 peaks in the left panel of Fig 1 corresponding to coordinations $L=1$ (dangling bond) to $L=7$. These results have in fact been obtained earlier via different routes [51]. A zoom into the low $x_{2}$ (large $\xi$ ) region reveals significant substructure which shows that correlation lengths of particles depend in fact also on coordinations of their neighbours (and on coordinations of next neighbours and so on).
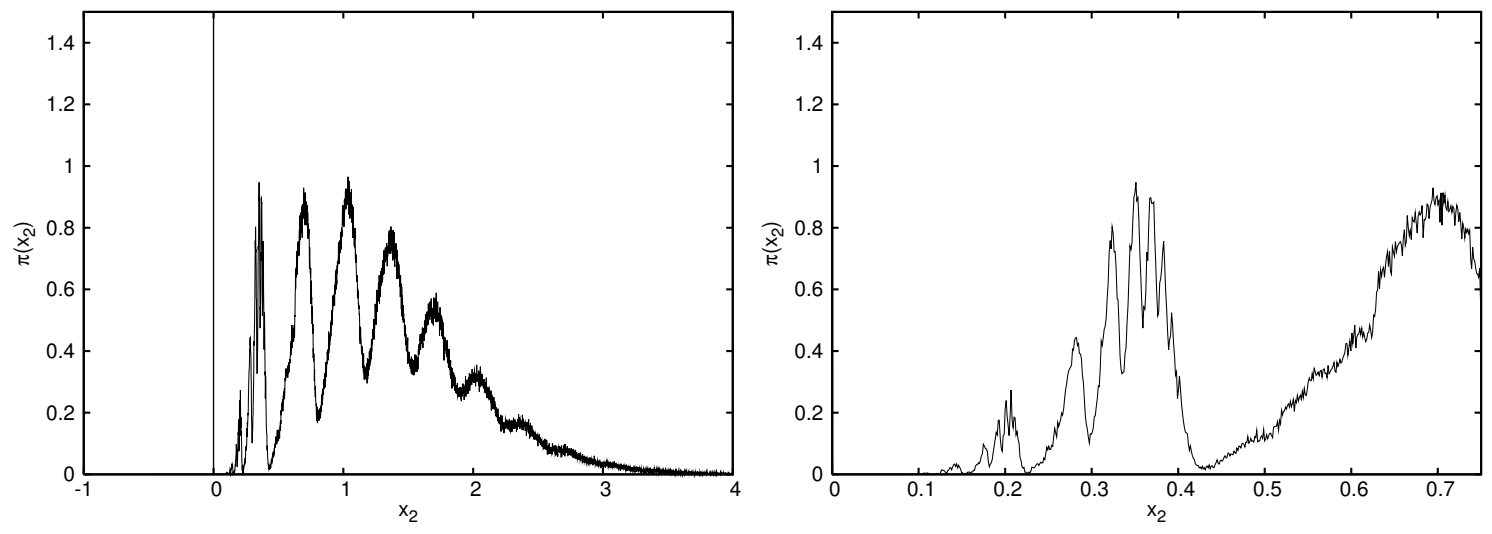

Figure 1: Distribution $\pi\left(x_{2}\right)$ for a harmonically coupled system with homogeneous coupling strengths $k_{i j} \equiv 1$ on a Poissonian random graph of average connectivity $C=4$; the inverse temperature is $\beta=1$. Left: full distribution; right: zoom into the low $x_{2}$ region. The $\delta$-peak at $x_{2}=0$ in the left panel is not drawn to scale; its total weight corresponds to the fraction of vertices not in the percolating cluster.

In Fig 2 we show analogous results for a graph with non-Poissonian degree distribution, assuming connectivities of the form $L=2+L^{\prime}$ with $L^{\prime}$ a Poissonian of mean 2. In effect, therefore, we do again have mean connectivity $C=4$. However, there are no longer isolated clusters of finite size.

For the non-Poissonian random graph, a comparison with the distribution of the expansion coefficient $x_{\text {eff,2 }}$ of the effective single site potentials in Fig 3 reveals that these ensembles while qualitatively similar — are indeed not identical. 
As a last result for the harmonic system, we look at the distribution the expansion coefficient $x_{\text {eff, } 2}$ for a system in which we have non-uniform coupling strengths in addition to locally varying connectivities. In the present case we took $k_{i j}$ uniformly distributed in $[0,1]$, and choose a nonPoissonian graph structure of with $C=4$ of the same type as before. Due to the variability in the $k_{i j}$, the peak structure in the $x_{\mathrm{eff}, 2}$-distribution is smeared, and it is no longer possible to associate a value of the localization length with a local connectivity, simply by looking at the distribution. However, the method of determining the distribution of effective single site potentials easily allows to monitor the contributions coming from sites with different connectivities, as illustrated in Fig. 4.

\section{$5 \quad$ Glassy Sytems with Anharmonicities}

The next model that we investigate is a random graph of coupled (scalar) degrees of freedom with a distribution $P\left(k_{i j}\right)$ of harmonic coupling strengths and fixed anharmonic coupling strength. Furthermore, we assume that there are non-random anharmonic single-site potentials. The interaction energy between connected vertices $(i, j)$ of the graph is taken to be of the form

$$
\phi_{i j}\left(u_{i}-u_{j}\right)=\frac{k_{i j}}{2}\left(u_{i}-u_{j}\right)^{2}+\lambda_{4}\left(u_{i}-u_{j}\right)^{4}
$$
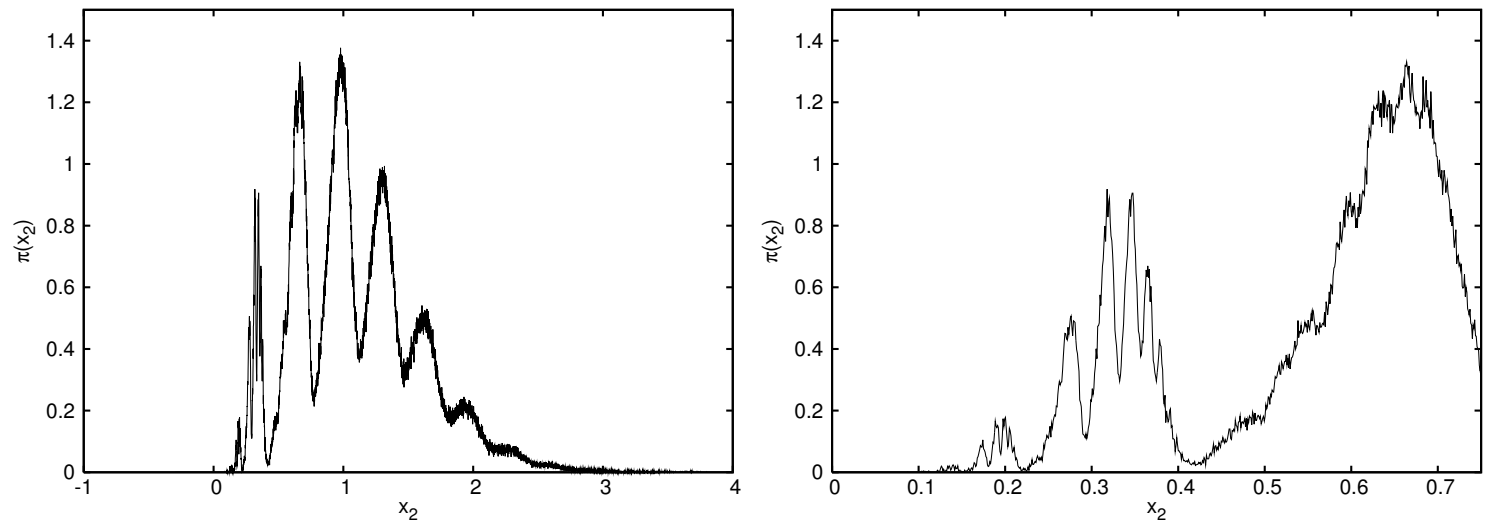

Figure 2: Distribution $\pi\left(x_{2}\right)$ for a harmonically coupled system with homogeneous coupling strengths $k_{i j} \equiv 1$ on a non-Poissonian random graph of average connectivity $C=4$ as described in the text; the inverse temperature is $\beta=1$. Left: full distribution; right: zoom into the low $x_{2}$ region. 


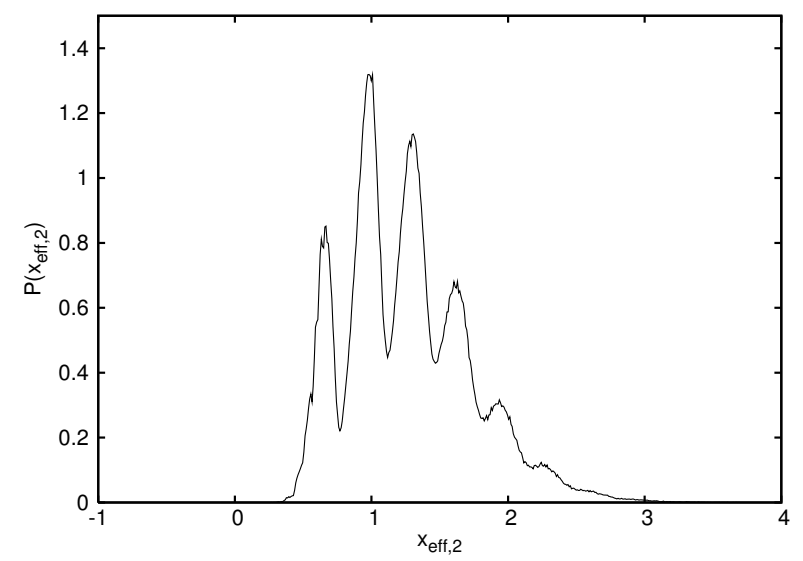

Figure 3: Distribution $P\left(x_{\mathrm{eff}, 2}\right)$ for a harmonically coupled system with homogeneous coupling strengths $k_{i j} \equiv 1$ on a non-Poissonian random graph of average connectivity $C=4$ as described in the text; the inverse temperature is $\beta=1$.

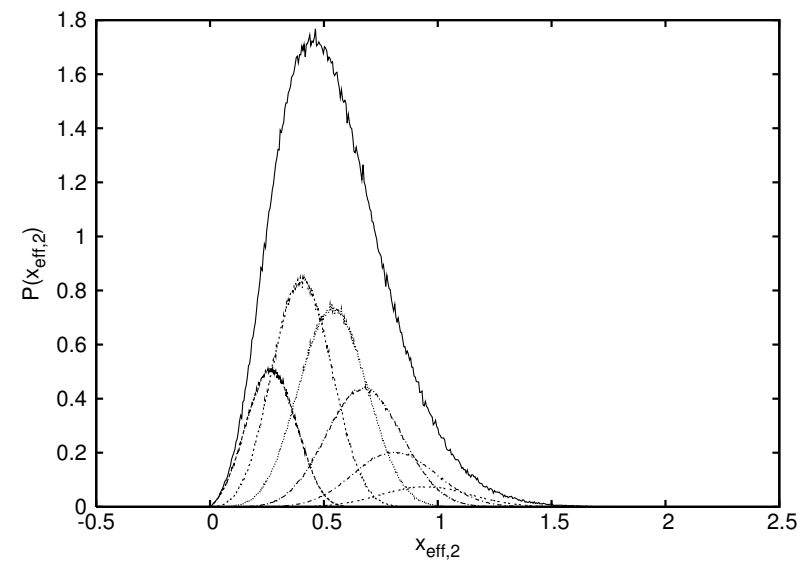

Figure 4: Distribution $P\left(x_{\mathrm{eff}, 2}\right)$ shown together with separate contributions to it coming from sites with connectivities $L=2,3, \ldots, 7$, for a harmonically coupled system with couplings $k_{i j} \sim$ $\mathcal{U}[0,1]$ on a non-Poissonian random graph of average connectivity $C=4$ as described in the text; the inverse temperature is $\beta=1$.

and we introduce a vertex-independent single-site potential

$$
V\left(u_{i}\right)=g_{4} u_{i}^{4}
$$

If the support of $P\left(k_{i j}\right)$ contains negative couplings, at least one of the quartic couplings $\lambda_{4}$ or 
$g_{4}$ must be positive for the system to be stable.

A fully connected variant of this model with $g_{4}>0$ and $\lambda_{4}=0$ was studied in [38], the corresponding model with $g_{4}=0$ and $\lambda_{4}>0$ in [39]. For the purposes of describing glassy low-temperature anomalies either form of stabilization by a quartic anharmonicity appeared to be acceptable. Differences between low-temperature properties of the two models were mainly in the details, although from a fundamental point of view the system with $g_{4}=0$ would of course be preferable, since $g_{4} \neq 0$ breaks translational invariance.

For the purposes of the present investigation we shall nevertheless stick to the local stabilizing potentials, i.e. choose $\lambda_{4}=0$ and $g_{4} \neq 0$, because this form of stabilization is numerically much easier to handle than its translationally invariant counterpart. We will turn to anharmonic systems with full translational invariance in a separate publication.

From the solutions for the distributions $\pi[\psi]$ and $\hat{\pi}[\hat{\psi}]$ of single-replica potentials one obtains a distribution of effective-single site potentials via (31).

Using an expansion of effective single-site potentials $V_{\text {eff }}(u)$ in terms of the modified Hermite polynomials used in Sec 4 to describe harmonic systems,

$$
V_{\mathrm{eff}}(u)=\sum_{\mu} x_{\mu} \varphi_{\mu}(u)
$$

the distribution of effective-single site potentials translates into a distribution of expansion coefficients $\left\{x_{\mu}\right\}$ (to simplify notation, we drop the subscript 'eff' on the expansion coefficients).

For systems with (quartic) anharmonicities, the solution of the fixed point equations (23), (24) involves distributions of single-replica potentials $\psi$ and $\hat{\psi}$ with support on potentials having anharmonicities of all orders. Any finitely truncated orthogonal function representation of these distributions would therefore only generate an approximate solution to the full problem (on top of the approximation implied in the RS assumption). It is therefore advisable to use the full functional approach (not least in order to check the quality of finite dimensional approximations). Below we therefore show distributions of some low order expansion coefficients as obtained in the functional approach and compare them with corresponding distributions obtained within a low-order truncation scheme.

We fix the energy scale of the system by setting $g_{4}=1$, and introduce frustration in the system by taking Gaussian distributed harmonic couplings, $k_{i j} \sim \mathcal{N}\left(0, \sigma_{k}\right)$. A system of this type then undergoes a transition from a symmetric high temperature phase, in which all effective single-site potentials respect the microscopic inversion symmetry of the original interaction energy $V_{\text {eff }}(u)=$ $V_{\text {eff }}(-u)$, to a glassy low-temperature phase, in which this symmetry is spontaneously broken, i.e. which is described by an ensemble of single-site potentials for which typically $V_{\text {eff }}(u) \neq V_{\text {eff }}(-u)$. This transition occurs at a temperature $T_{c}$ which depends on the strength $\sigma_{k}$ of the disorder (and of course on the connectivity distribution). Indeed, for a combination of quadratic and 
quartic potentials as chosen in the present setup, dimensional analysis reveals that the critical condition should have a parameter dependence of the form

$$
T_{c}\left(\sigma_{k}, g_{4}\right)=\frac{\sigma_{k}^{2}}{g_{4}} T_{c}(1,1) \text {. }
$$

as already found for the fully connected system [39]. Here the value of $T_{c}(1,1)$ still depends, of course, on the connectivity distribution. E.g., for the system with the non-Poissonian connectivity distribution with average degree $C=4$ looked at earlier, we determined $T_{c}(1,1)=$ $0.932 \pm 0.005 \Leftrightarrow \beta_{c}(1,1) \simeq 1.073$, and we verified the scaling (53) with high precision within the full functional treatment of the self-consistency equations. It is important to note, however, that approximate treatments, such as the low-order orthogonal function approximation procedure, need not respect this scaling of $T_{c}\left(\sigma_{k}, g_{4}\right)$, though we found them to be not far off.

The transition to glassy order at low temperatures is continuous in the sense that widths of the distributions of the expansion coefficients $x_{1}, x_{3}, x_{5}, \ldots$ coupling to anti-symmetric functions go to zero continuously as the transition temperature is approached from below. Fig 5 illustrates this for a system with a non-Poissonian connectivity distribution of the type used in Sec 4 with $C=4$, and $\sigma_{k}=1$. If an orthogonal function representation of the single-replica functions in terms of Hermite polynomials, truncated at order four is used instead of the full functional approach, we find the order of the transition to be unchanged, though the transition point is shifted to a sligthly higher temperature, $T_{c}(1,1) \simeq 1.043 \Leftrightarrow \beta_{c}(1,1) \simeq 0.959$ (differing from the exact value by approximately $3 \%$ ).

We note that the population-dynamics algorithm exhibits critical slowing down as the transition is approached. In Fig 5. It is thererfore crucial to use sufficiently long runs and small temperature steps close to the transition to ensure that the algorithm has properly converged, before taking measurements.

Fig 6 shows distributions of expansion coefficients for a system with the non-Poissonian connectivity distribution used before, at $\sigma_{k}=1.1$, for $T=1$ and $T=0$, and compares distributions obtained from a full functional solution with those computed in an orthogonal function representation using Hermite polynomials that is truncated at order 4. Semilogarithmic plots are used to exhibit the behaviour in the tails of the distributions. At $T=1$, it turns out that the distributions of odd coefficients $x_{1}, x_{3}, \ldots$ appear to be affected by the low order truncation in a slightly stronger way than the distributions of even oder coefficients, and distributions of low order odd coefficients appear to be systematically slightly wider when computed via a truncated expansion approach; the main reason seems to be that the low order truncation has slightly higher critical temperature and with identical parameter settings, the system appears to be deeper in the glassy phase when described within the low order truncation scheme than when described in terms of full functional self-consistency. The distributions of the even coefficients, i.e. $x_{2}, x_{4} \ldots$ computed in an approximation scheme truncated order 4 appear to be less strongly affected by the truncation scheme. 


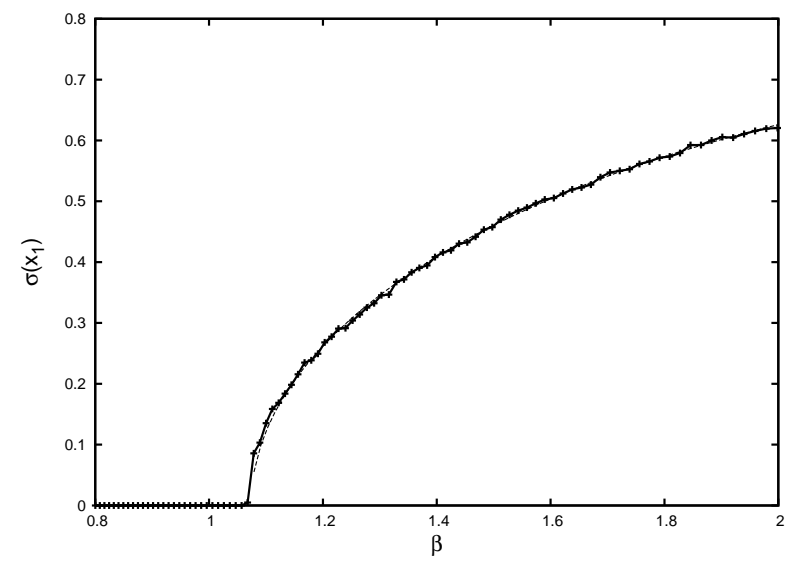

Figure 5: Width $\sigma\left(x_{1}\right)$ of the distribution of the first expansion coefficient $x_{1}$ in in the ensemble of effective single-site potentials. The width goes to zero with a square-root singularity at $\beta_{c} \simeq$ 1.073. A fit displaying a square-root singularity with analytic corrections describes the data very well over a wide range of inverse temperatures. For this system $\sigma_{k}=1$.

It should be noted that there are strong correlations between the expansion coefficients that can be exhibited, e.g. by computing their correlation coefficients or by inspecting scatterplots of individual realizations. Fig. 7 shows some examples. For the system under investigation we found the $\left(x_{2 k-1}, x_{2 \ell}\right)$ scatterplots to be similar up to scaling and symmetry transformations; analogous similarities exist in $\left(x_{2 k}, x_{2 \ell}\right)$-families at fixed $k$.

Differences between the full functional solutions and those obtained within a low-order expansion become rapidly less pronounced when moving away from the transition temperature. This is particularly clear in the the zero-temperature limit where an orthogonal function representation truncated at order 4 describes the results remarkably well, as seen in the wider set of curves also displayed in Fig 6. This might have been anticipated, as anharmonicities typically play a less significant role at low temperatures.

Another feature that deserves mention is that the distribution $P\left(x_{2}\right)$ of the second order coefficient his hardly temperature dependent at all (e.g., the zero-temperature limit of this distribution is virtually indistinguishable from the one at $T=1$ (as seen in the second panel of Fig 6) whereas all other distributions do show a noticeable variation with temperature.

Fig 8 gives the phase diagram of the system, showing the transition temperature as a function of the strength $\sigma_{k}$ of the disorder in the harmonic coupling constants.

In Fig 9 we show free energy, internal energy, entropy and specific heat as functions of temperature, for $\sigma_{k}=1$ and $g_{4}=1$, so that $T_{c} \simeq 0.93$. The specific heat is obtained through a numerical differentiation from the internal energy, and becomes fairly noisy for temperatures 

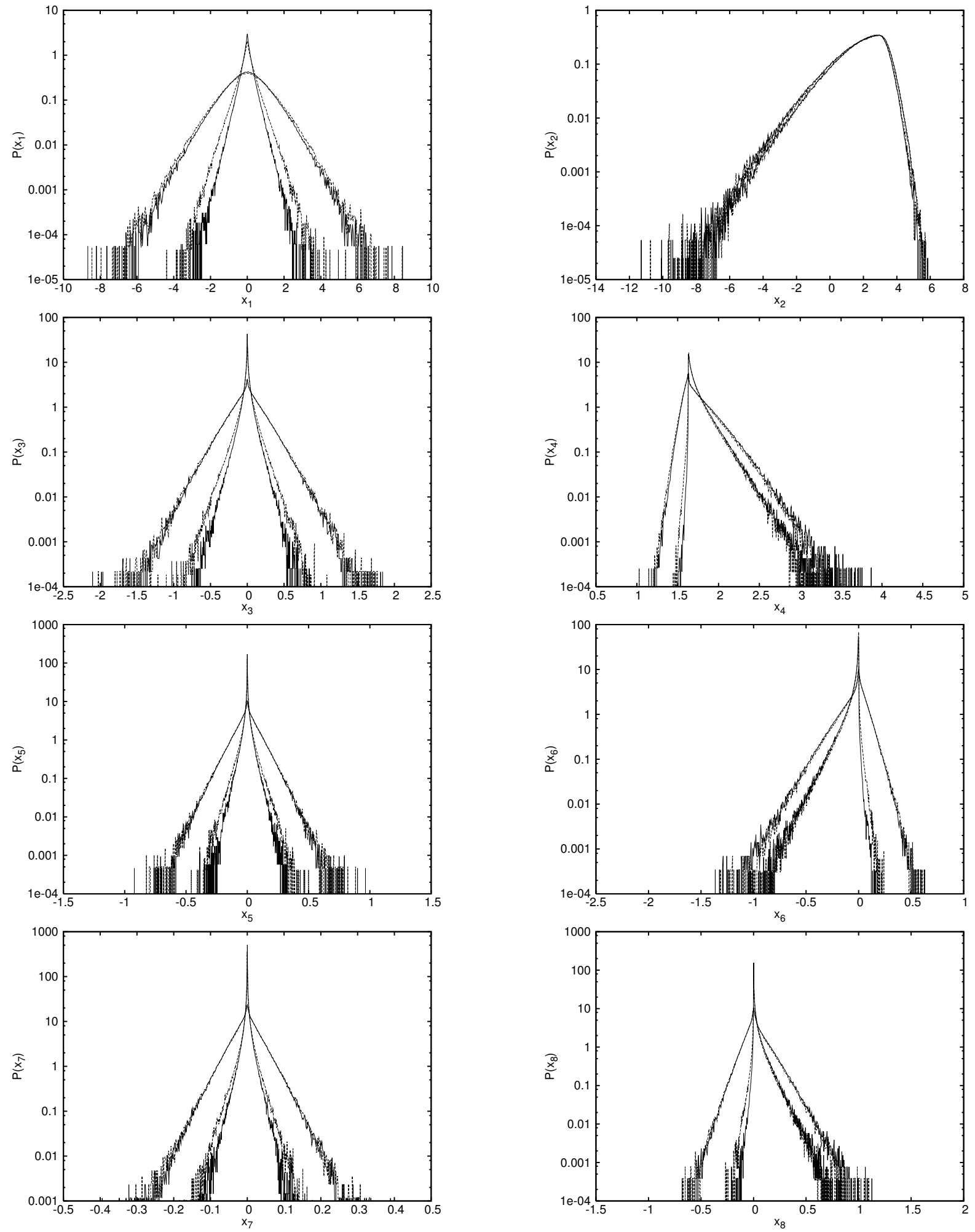

Figure 6: Distributions of low-order expansion coefficient $x_{\mu}, \mu=1, \ldots, 8$ in in the ensemble of effective single-site potentials for $\sigma_{k}=1.1$ at $T=1$ (narrower set of curves) and $T=0$ (wider set of curves). Solutions obtained within the full functional approach (full lines) are compared with those obtained by truncating expansions of single-replica potentials in terms of Hermite polynomials at order 4 . (dashed lines) 

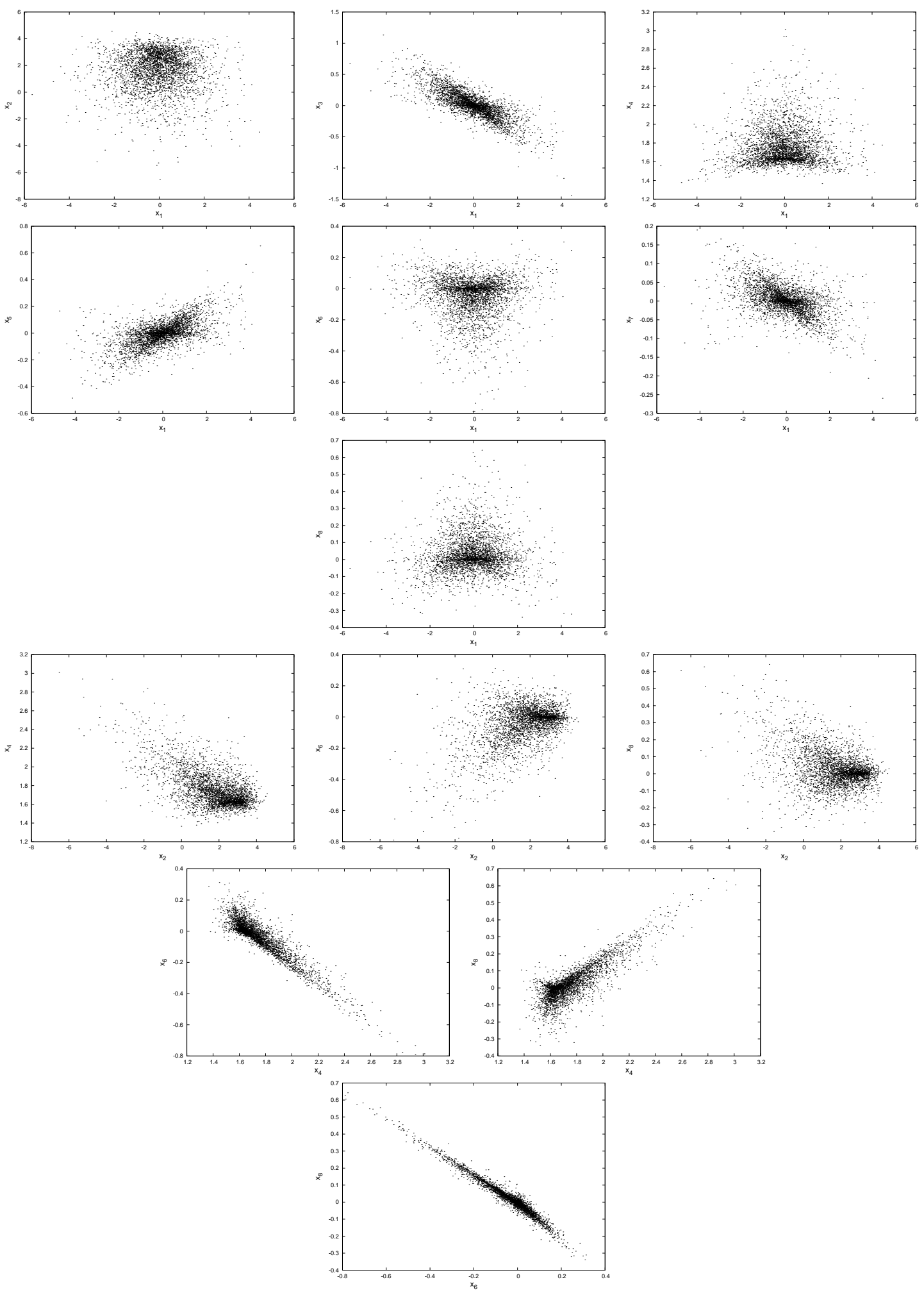

Figure 7: Scatterplots showing 2 dimensional projections of vectors of expansion coefficients for realizations of effective potentials. First group of panels $\left(x_{1}, x_{2}\right),\left(x_{1}, x_{3}\right), \ldots\left(x_{1}, x_{8}\right)$, second group $\left(x_{2}, x_{4}\right),\left(x_{2}, x_{6}\right),\left(x_{2}, x_{8}\right)$, third group $\left(x_{4}, x_{6}\right)$ and $\left(x_{4}, x_{8}\right)$, and last panel $\left(x_{6}, x_{8}\right)$. 


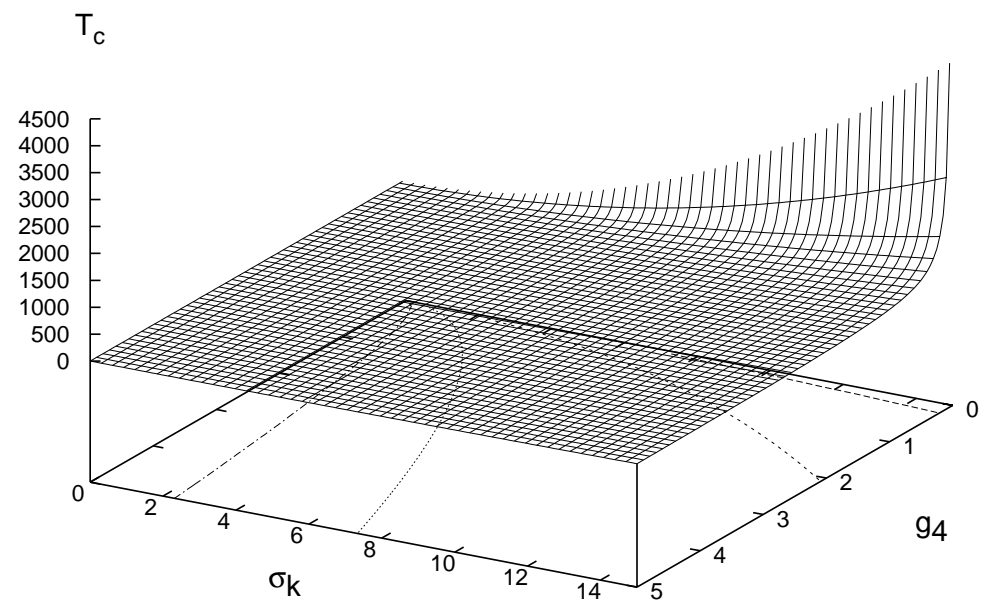

Figure 8: Transition temperature of the anharmonic system as a function of the standard deviation $\sigma_{k}$ of the random harmonic coupling, and the strength $g_{4}$ of the quartic stabilizing potential. Iso-lines for $T_{c}=0.1, T_{c}=1, T_{c}=10, T_{c}=100$, and $T_{c}=1000$ are also shown in the $\left(\sigma_{k}, g_{4}\right)$-plane

below $T \simeq 0.5$. Notice that the entropy becomes negative for $T \lesssim 0.31$, i.e. well inside the glassy phase. As our system has continous degrees of freedom, no strong conclusions can be drawn from this observation, however. It must be pointed out, though, that we expect replica symmetry to be broken throughout the low-temperature phase, and that our results require further checks, e.g. through simulations.

Finally, a word on numerics may perhaps be in order. It turns out that a good compromise between control and efficiency in evaluating functions like $\hat{\Psi}[\psi, \phi]$ on a sufficiently dense grid of points $\boldsymbol{v}$ via (27) as required for the population dynamics algorithm, is to use Gaussian quadratures with sufficiently many grid points. Further acceleration of numerical procedures is possible by evaluating functions initially on coarser grids, and obtaining their values on the fine grids required for further processing via suitable interpolation algorithms. 

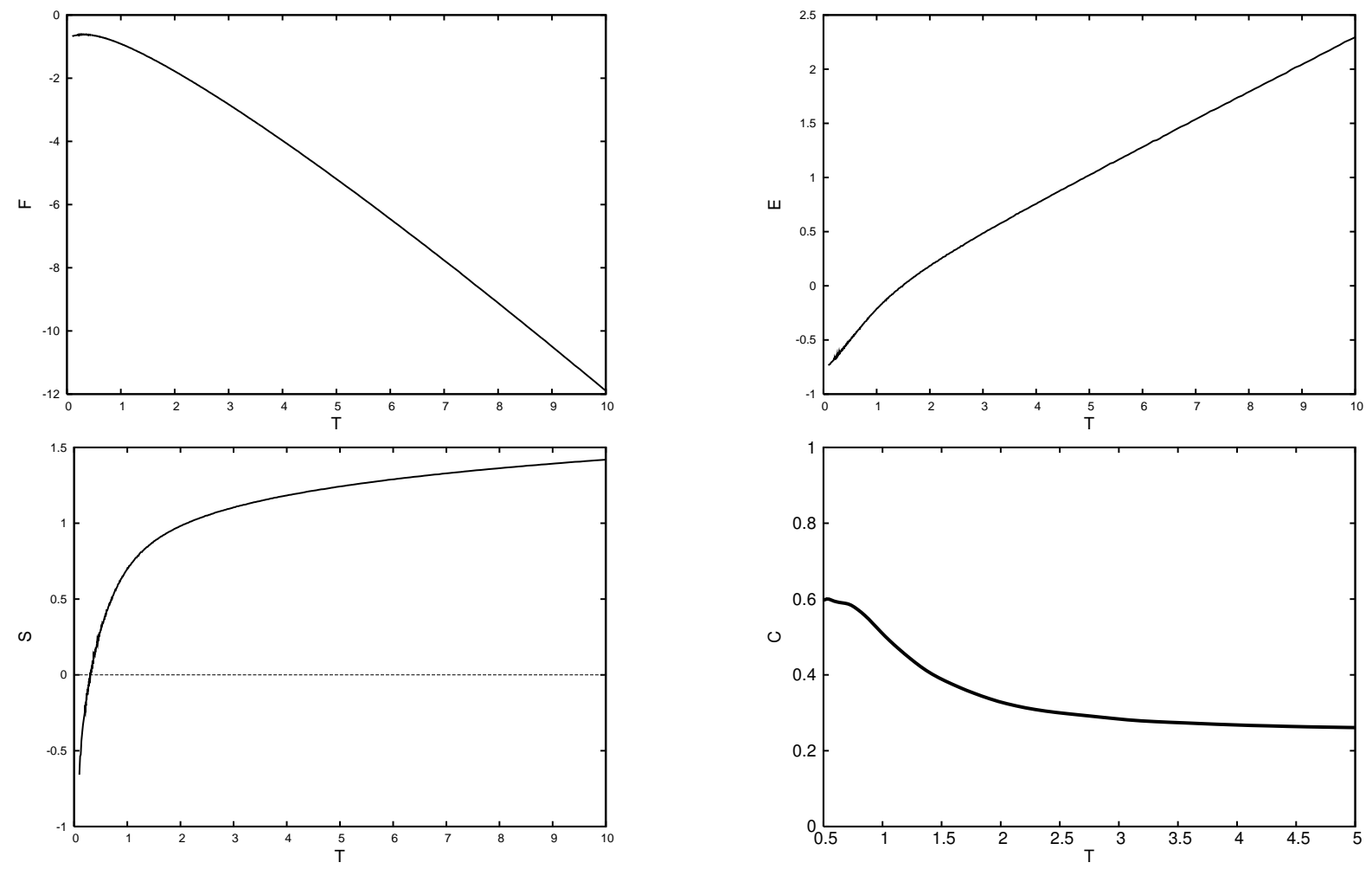

Figure 9: Thermodynamic functions: free Energy $f$ (first panel), internal energy $E$ (second panel), entropy $S$ (third panel), and specific heat $C$, (fourth panel).

\section{Conclusion}

We have introduced and solved a wide class of random models with finite-connectivity for the description of amorphous solid phases. The description is in terms of deviations of particle positions from a set of reference positions. The framework is kept general in the sense that we do not need not restrict the nature of the pair potential, nor the nature of the connectivity distribution, as long as they allow taking a meaningful thermodynamic limit. By varying the connectivity distributions or the nature of interaction potentials we expect to be able to describe, at least in a schematic manner, a fairly wide spectrum of different physical systems.

In the present paper we have looked in detail only at harmonically coupled systems with scalar degrees of freedom, thought to provide an appropriate description of systems in their gel-phase, and at a model with local quartic anharmonicities which could be used to describe glassy lowtemperature properties. The transition to the phase with frozen glassy order in the model con- 
sidered here is continuous, as in the corresponding fully connected variant. In this respect, the description of glass-transition physics within this model must still be regarded as deficient. This may or may not be due to the fact that the low-order expansions of the interaction energy chosen in the present paper can be considered as adequate description of the physics only in the limit of low temperatures.

One promising possibility to improve the description of glass-transition physics within the present model-class would be to look at models with non-confining interaction potentials. This would inter alia allow obtaining more adequate descriptions of high temperature phases, as phases with vanishing inverse localization lengths (delocalized particles). It remains to be seen whether this modification could put our model into the class for which glass-transition physics would be described by the succession of dynamic freezing and entropy-crisis scenario thought to be appropriate for structural glasses.

Other problems we might be able to shed some analytic light on are correlations between coordination and effective potential statistics, or - by looking at mixtures of different particle species - the correlations between particle species and their enhanced or depressed role in the formation of tunneling centres in amorphous systems.

Another open question is related to the fact that the present models - unlike their fully connected counterparts - are not completely described in terms of ensembles of effective noninteracting single-site problems. The two-particle contribution appearing in the free energy is a consequence of the fact that finite connectivity systems of the type considered here would no longer support simple single particle excitations. It is conceivable that the two-particle contribution would allow an interpretation similar to the 'heat-bath' of phonons acting on tunnelling systems that is introduced in an ad-hoc manner into phenomenological models of glassy lowtemperature anomalies $[28,29,55]$.

We intend to look into some of these questions in future publications.

Acknowledgements It is a pleasure to thank ACC Coolen, J Hatchett, I Perez-Castillo, and $\mathrm{P}$ Sollich for many illuminating discussions and helpful remarks. 


\section{A Details of the Replica Analysis}

\section{A.1 Averaging the Replicated Partition Function}

Given the distribution (4) of connectivity matrices, the average (7) of the replicated partition function has the following structure

$$
\left\langle Z_{N}^{n}\right\rangle_{\mathbf{C}, \phi}=\frac{\mathcal{T}}{\mathcal{N}} \equiv \frac{\left\langle\prod_{(i, j)} \operatorname{Tr}_{c_{i j}} p_{C}\left(c_{i j}\right) \delta_{c_{i j}, c_{j i}} \prod_{i} \delta_{L_{i}, \sum_{j(\neq i)} c_{i j}} Z_{N}^{n}\right\rangle_{\phi}}{\prod_{(i, j)} \operatorname{Tr}_{c_{i j}} p_{C}\left(c_{i j}\right) \delta_{c_{i j}, c_{j i}} \prod_{i} \delta_{L_{i}, \sum_{j(\neq i)} c_{i j}}} .
$$

Note that both the $c_{i j}$-summations and the average over local pair potentials $\phi_{i j}$, factor w.r.t the pairs $(i, j)$.

It should be mentioned that other, equivalent ways of introducing the ensemble of connectivity matrices are possible. E.g. one could include summations over all possible assignments of local connectivities $\left\{L_{i}\right\}$ compatible with a given $P_{C}(L)$ in both $\mathcal{T}$ and $\mathcal{N}$ in (54), without changing final results, as this would just create contributions coming from the entropy of the connectivity distribution in $\mathcal{T}$ and $\mathcal{N}$, which cancel. Similarly, one could omit including the single bond distributions $p_{C}\left(c_{i j}\right)$ in $\mathcal{T}$ and $\mathcal{N}$, as long as the constraints required by the desired connectivity contribution $P_{C}(L)$ are kept - at the cost of considerably complicating the algebra, and without changing final results. In what follows, we stick to the version embodied in (54).

To evaluate (54), one uses integral representations of the form (9) to express the constraints on the local coordinations $L_{i}$. The normalization constant $\mathcal{N}$ in (54) is then transformed into an integral expression, which can be evaluated via the saddle point method, along the following transparent sequence of steps

$$
\begin{aligned}
\mathcal{N} & =\prod_{(i, j)} \operatorname{Tr}_{c_{i j}} p_{C}\left(c_{i j}\right) \delta_{c_{i j}, c_{j i}} \prod_{i} \oint \frac{\mathrm{d} z_{i}}{2 \pi \mathrm{i} z_{i}} z_{i}^{\left(\sum_{j(\neq i)} c_{i j}-L_{i}\right)} \\
& =\prod_{i} \oint \frac{\mathrm{d} z_{i}}{2 \pi \mathrm{i} z_{i}} z_{i}^{-L_{i}} \prod_{(i, j)} \operatorname{Tr}_{c_{i j}} p_{C}\left(c_{i j}\right)\left(z_{i} z_{j}\right)^{c_{i j}} \\
& \simeq \prod_{i} \oint \frac{\mathrm{d} z_{i}}{2 \pi \mathrm{i} z_{i}} z_{i}^{-L_{i}} \mathrm{e}^{\left(\frac{C}{2 N} \sum_{i, j}\left(z_{i} z_{j}-1\right)\right)} \\
& =\int \frac{\mathrm{d} \hat{\rho}_{0} \mathrm{~d} \rho_{0}}{2 \pi / N} \exp \left(N\left\{\frac{C}{2}\left(\rho_{0}^{2}-1\right)-\hat{\rho}_{0} \rho_{0}\right\}\right) \prod_{i} \oint \frac{\mathrm{d} z_{i}}{2 \pi \mathrm{i} z_{i}} z_{i}^{-L_{i}} \mathrm{e}^{\hat{\rho}_{0} z_{i}} \\
& =\int \frac{\mathrm{d} \hat{\rho}_{0} \mathrm{~d} \rho_{0}}{2 \pi / N} \exp \left(N\left\{\frac{C}{2}\left(\rho_{0}^{2}-1\right)-\hat{\rho}_{0} \rho_{0}+\frac{1}{N} \sum_{i} \ln \left(\frac{\hat{\rho}_{0}^{L_{i}}}{L_{i} !}\right)\right\}\right)
\end{aligned}
$$

Here, we used large $N$ asymptotics to re-exponentiate the $c_{i j}$ averages in line 2, and introduced $\rho_{0} \equiv \frac{1}{N} \sum_{i} z_{i}$, enforced by the conjugate $\hat{\rho}_{0}$, to obtain an expression that can be evaluated by 
the saddle point method, and finally exploited the fact that (9) entails

$$
\oint \frac{\mathrm{d} z}{2 \pi \mathrm{i} z} z^{-L} f(z)=\left.\frac{1}{L !} \frac{\partial^{L} f(z)}{\partial z^{L}}\right|_{z=0} .
$$

As the $L_{i}$ are distributed according to $P_{C}(L)$, the last contribution in the exponent in (55) can be expressed as the $L$-average $\sum_{L} P_{C}(L) \ln \left(\frac{\hat{\rho}_{0}^{L}}{L !}\right)$ by the law of large numbers. In the saddle point of $(55)$, i.e. $\left(\rho_{0}=1, \hat{\rho}_{0}=C\right)$ we finally have:

$$
\mathcal{N} \sim \exp \left(N\left\{\sum_{L} P_{C}(L) \ln \left(\frac{C^{L}}{L !}\right)-C\right\}\right)
$$

Given any typical realization of local potentials $\left\{V_{i}, i=1, . ., N\right\}$, and using the shorthands (8) for the replicated bond and site weights we have for nominator $\mathcal{T}$ in (54), following similar lines of reasoning:

$$
\begin{aligned}
\mathcal{T} & =\prod_{i} \int \mathrm{d} \tilde{\boldsymbol{u}}_{i} \mathcal{U}_{s}\left(\tilde{\boldsymbol{u}}_{i}, V_{i}\right) \prod_{(i, j)} \operatorname{Tr}_{c_{i j}} p_{C}\left(c_{i j}\right) \delta_{c_{i j}, c_{j i}}\left\langle\left(\mathcal{U}_{b}\left(\tilde{\boldsymbol{u}}_{i}, \tilde{\boldsymbol{u}}_{j}, \phi\right)\right)^{c_{i j}}\right\rangle_{\phi} \prod_{i} \delta_{L_{i}, \sum_{j(\neq i)} c_{i j}} \\
& \simeq \prod_{i} \int \mathrm{d} \tilde{\boldsymbol{u}}_{i} \mathcal{U}_{s}\left(\tilde{\boldsymbol{u}}_{i}, V_{i}\right) \oint \frac{\mathrm{d} z_{i}}{2 \pi \mathrm{i} z_{i}} z_{i}^{-L_{i}} \mathrm{e}^{\frac{C}{2 N} \sum_{i, j} z_{i} z_{j}\left\langle\mathcal{U}_{b}\left(\tilde{\boldsymbol{u}}_{i}, \tilde{\boldsymbol{u}}_{j}, \phi\right)\right\rangle_{\phi}-\frac{C N}{2}} \\
& =\int \mathcal{D} \rho \mathcal{D} \hat{\rho} \exp \left(N\left\{\frac{C}{2}\left(G_{b}[\rho]-1\right)-G_{m}[\rho, \hat{\rho}]\right\}\right) \prod \prod_{i} \int \mathrm{d} \tilde{\boldsymbol{u}}_{i} \mathcal{U}_{s}\left(\tilde{\boldsymbol{u}}_{i}, V_{i}\right) \oint \frac{\mathrm{d} z_{i}}{2 \pi \mathrm{i} z_{i}} z_{i}^{-L_{i}} \mathrm{e}^{z_{i} \hat{\rho}\left(\tilde{\boldsymbol{u}}_{i}\right)} \\
& \left.=\int \mathcal{D} \rho \mathcal{D} \hat{\rho} \exp \left(N\left\{\frac{C}{2}\left(G_{b}[\rho]-1\right)-G_{m}[\rho, \hat{\rho}]+G_{s}[\hat{\rho}]\right]\right\}\right)
\end{aligned}
$$

The definition (10) of the replica density enforced by a conjugate $\hat{\rho}(\tilde{\boldsymbol{u}})$ was used to express the result in terms of a path-integral that can be evaluated by the saddle point method.

The three functionals appearing in (58) are given as

$$
\begin{aligned}
G_{b}[\rho] & =\left\langle\int \mathrm{d} \tilde{\boldsymbol{u}} \mathrm{d} \tilde{\boldsymbol{v}} \rho(\tilde{\boldsymbol{u}}) \mathcal{U}_{b}(\tilde{\boldsymbol{u}}, \tilde{\boldsymbol{v}}, \phi) \rho(\tilde{\boldsymbol{v}})\right\rangle_{\phi} \\
G_{m}[\rho, \hat{\rho}] & =\int \mathrm{d} \tilde{\boldsymbol{u}} \hat{\rho}(\tilde{\boldsymbol{u}}) \rho(\tilde{\boldsymbol{u}}) \\
G_{s}[\hat{\rho}] & =\sum_{L} P_{C}(L)\left\langle\ln \int \mathrm{d} \tilde{\boldsymbol{u}} \frac{\hat{\rho}^{L}(\tilde{\boldsymbol{u}})}{L !} \mathcal{U}_{s}(\tilde{\boldsymbol{u}}, V)\right\rangle_{V}
\end{aligned}
$$

where the law of large numbers is used to write $G_{s}$ as an average over the site disorder (in the $L_{i}$ and the $V_{i}$ ). 


\section{A.2 Replica Symmetry}

We proceed by making an ansatz for $\rho$ and $\hat{\rho}$ which amounts to assuming unbroken replica symmetry (RS):

$$
\begin{aligned}
& \rho(\tilde{\boldsymbol{u}})=\int \mathcal{D} \psi \pi[\psi] \prod_{a} \frac{\exp \left[-\beta \psi\left(\boldsymbol{u}^{a}\right)\right]}{Z[\psi]} \\
& \hat{\rho}(\tilde{\boldsymbol{u}})=\int \mathcal{D} \hat{\psi} \hat{\pi}[\hat{\psi}] \prod_{a} \frac{\exp \left[-\beta \hat{\psi}\left(\boldsymbol{u}^{a}\right)\right]}{Z[\hat{\psi}]},
\end{aligned}
$$

where $\pi$ and $\hat{\pi}$ are functionals over the space of single site potentials and $Z[\psi]$ and $Z[\hat{\psi}]$ are defined via (17). We then obtain:

$$
\begin{aligned}
G_{b}[\rho] & \simeq \rho_{0}^{2}+n \int \mathcal{D} \pi\left[\psi_{1}\right] \mathcal{D} \pi\left[\psi_{2}\right]\left\langle\ln \left(\frac{Z_{2}\left[\psi_{1}, \psi_{2}, \phi\right]}{Z\left[\psi_{1}\right] Z\left[\psi_{2}\right]}\right)\right\rangle_{\phi} \\
G_{m}[\rho, \hat{\rho}] & \simeq \rho_{0} \hat{\rho}_{0}+n \int \mathcal{D} \pi[\psi] \mathcal{D} \hat{\pi}[\hat{\psi}] \ln \left(\frac{Z[\psi+\hat{\psi}]}{Z[\psi] Z[\hat{\psi}]}\right) \\
G_{s}[\hat{\rho}] & \simeq \sum_{L} P_{C}(L)\left(\ln \left(\frac{\hat{\rho}_{0}^{L}}{L !}\right)+n \int\{\mathcal{D} \hat{\pi}\}_{L}\left\langle\ln \left(\frac{Z\left[\sum_{\ell}^{L} \hat{\psi}_{\ell}+V\right]}{\prod_{\ell=1}^{L} Z\left[\hat{\psi}_{\ell}\right]}\right)\right\rangle_{V}\right)
\end{aligned}
$$

where the definition (19) of $Z_{2}$, the convention $\{\mathcal{D} \hat{\pi}\}_{L} \equiv \prod_{\ell}^{L} \mathcal{D} \hat{\pi}\left[\hat{\psi}_{\ell}\right]$, and the abbreviations $\rho_{0} \equiv \int \mathcal{D} \pi[\psi]$, and $\hat{\rho}_{0} \equiv \int \mathcal{D} \hat{\pi}[\hat{\psi}]$ for full integrals over the functionals $\pi$ and $\hat{\pi}$ have been used. We first solve the saddle point equations to $\mathcal{O}(1)=\mathcal{O}\left(n^{0}\right)$ in $n$, to obtain $\rho_{0}=1$, and $\hat{\rho}_{0}=C$. This entails that the $\mathcal{O}\left(n^{0}\right)$ contributions to $\mathcal{T}$ cancel with $\mathcal{N}$, and we finally obtain

$$
\left\langle Z_{N}^{n}\right\rangle_{\mathbf{C}, \phi} \sim \int \mathcal{D} \pi \mathcal{D} \hat{\pi} \exp \left(n N\left\{\frac{C}{2} \mathcal{G}_{b}[\pi]-C \mathcal{G}_{m}[\pi, \hat{\pi}]+\sum_{L} P_{C}(L) \mathcal{G}_{s, L}[\hat{\pi}]\right\}\right),
$$

in which $\pi$ and $\hat{\pi}$ are now normalised functionals, and where

$$
\begin{aligned}
\mathcal{G}_{b}[\pi] & \simeq \int \mathcal{D} \pi\left[\psi_{1}\right] \mathcal{D} \pi\left[\psi_{2}\right]\left\langle\ln \left(\frac{Z_{2}\left[\psi_{1}, \psi_{2}, \phi\right]}{Z\left[\psi_{1}\right] Z\left[\psi_{2}\right]}\right)\right\rangle_{\phi}, \\
\mathcal{G}_{m}[\pi, \hat{\pi}] & \simeq \int \mathcal{D} \pi[\psi] \mathcal{D} \hat{\pi}[\hat{\psi}] \ln \left(\frac{Z[\psi+\hat{\psi}]}{Z[\psi] Z[\hat{\psi}]}\right), \\
\mathcal{G}_{s, L}[\hat{\pi}] & \simeq \int\{\mathcal{D} \hat{\pi}\}_{L}\left\langle\ln \left(\frac{Z\left[\sum_{\ell=1}^{L} \hat{\psi}_{\ell}+V\right]}{\prod_{\ell=1}^{L} Z\left[\hat{\psi}_{\ell}\right]}\right)\right\rangle_{V} .
\end{aligned}
$$

The saddle point equations w.r.t. the normalised $\pi, \hat{\pi}$ can then be expressed in the form (23) and (24) as given in Sect 3.1. 


\section{A.3 Breaking Replica Symmetry}

It is expected that replica symmetry will be broken in phases with frozen glassy order. We will in the present paper not go on to solve our models assuming phases with broken replica symmetry. However we would here like to present at least the ansatz which would describe phases with one level of replica symmetry breaking in the spirit of Parisi' hierarchical scheme. It is based on grouping the $n$ replica in $n / m$ blocks containing $m$ replica each, and assuming symmetry of the solution w.r.t. permutations of replica within blocks though not between blocks. This would lead to

$$
\begin{aligned}
& \rho(\tilde{\boldsymbol{u}})=\int \mathcal{D} \pi \mathcal{P}[\pi] \prod_{k=1}^{n / m} \int \mathcal{D} \psi_{k} \pi\left[\psi_{k}\right] \prod_{a=(k-1) m+1}^{k m} \frac{\exp \left[-\beta \psi_{k}\left(\boldsymbol{u}^{a}\right)\right]}{Z\left[\psi_{k}\right]} \\
& \hat{\rho}(\tilde{\boldsymbol{u}})=\int \mathcal{D} \hat{\pi} \hat{\mathcal{P}}[\hat{\pi}] \prod_{k=1}^{n / m} \int \mathcal{D} \hat{\psi}_{k} \hat{\pi}\left[\hat{\psi}_{k}\right] \prod_{a=(k-1) m+1}^{k m} \frac{\exp \left[-\beta \hat{\psi}_{k}\left(\boldsymbol{u}^{a}\right)\right]}{Z\left[\hat{\psi}_{k}\right]},
\end{aligned}
$$

as ansatz for the replica-density (10) and the conjugate required to enforce its definition, respectively. I.e., we get a functional superposition of products of block-replica densities, each of which a functional superposition of products of single-replica Gibbs distributions over the replica within a block.

This ansats would again allow to formulate the $n \rightarrow 0$-limit of the theory, and would lead to fixed point equations for the weight functions $\mathcal{P}, \pi, \hat{\mathcal{P}}$ and $\hat{\pi}$ that can be cast into a form which would admit a solution in terms of a stochastic population-based algorithm — an algorithm, however, which requires to maintain an ensemble of populations of the type used in the RS

version of the theory. In addition, there is the stationarity requirement on the Free Energy with respect to the partitioning parameter $m$. The numerical effort required to solve this problem will be considerable.

\section{B Free Energy, Thermodynamic Functions}

The replica symmetric expression of the free energy as a funcional of $\pi$ and $\hat{\pi}$ obtained from (18)-(22) initially gives

$$
\begin{aligned}
-\beta f(\beta)= & \frac{C}{2} \int \mathcal{D} \pi[\psi] \mathcal{D} \pi\left[\psi^{\prime}\right]\left\langle\ln \left[\frac{Z_{2}\left[\psi, \psi^{\prime}, \phi\right]}{Z[\psi] Z\left[\psi^{\prime}\right]}\right]\right\rangle_{\phi}-C \int \mathcal{D} \pi[\psi] \mathcal{D} \hat{\pi}[\hat{\psi}] \ln \left[\frac{Z[\psi+\hat{\psi}]}{Z[\psi] \hat{Z}[\hat{\psi}]}\right] \\
& +\sum_{L} P_{C}(L) \int\{\mathcal{D} \hat{\pi}\}_{L}\left\langle\ln \left[\frac{Z\left[\sum_{\ell=1}^{L} \hat{\psi}_{\ell}+V\right]}{\prod_{\ell=1}^{L} \hat{Z}\left[\hat{\psi}_{\ell}\right]}\right]\right\rangle_{V},
\end{aligned}
$$


in which $\pi$ and $\hat{\pi}$ are taken to be solutions of the fixed-point equations.

Replacing logarithms of products (quotients) by sums (differences) of logaritms, and recalling and $\langle L\rangle=C$, we first get

$$
\begin{aligned}
-\beta f(\beta)= & \frac{C}{2} \int \mathcal{D} \pi[\psi] \mathcal{D} \pi\left[\psi^{\prime}\right]\left\langle\ln \left[Z_{2}\left[\psi, \psi^{\prime}, \phi\right]\right]\right\rangle_{\phi}-C \int \mathcal{D} \pi[\psi] \mathcal{D} \hat{\pi}[\hat{\psi}] \ln [Z[\psi+\hat{\psi}]] \\
& +\sum_{L} P_{C}(L) \int\{\mathcal{D} \hat{\pi}\}_{L}\left\langle\ln \left[Z\left[\sum_{\ell=1}^{L} \hat{\psi}_{\ell}+V\right]\right]\right\rangle_{V} .
\end{aligned}
$$

In a second step, we express $\hat{\pi}[\hat{\psi}]$ appearing in the second integral by an integral over $\pi[\psi]$ via (23) to get

$$
\begin{aligned}
\int \mathcal{D} \pi[\psi] \mathcal{D} \hat{\pi}[\hat{\psi}] \ln [Z[\psi+\hat{\psi}]] & =\int \mathcal{D} \pi[\psi] \mathcal{D} \pi\left[\psi^{\prime}\right] \int \mathcal{D} \hat{\psi}\left\langle\delta\left[\hat{\psi}-\hat{\Psi}\left[\psi^{\prime}, \phi\right]\right]\right\rangle_{\phi} \ln [Z[\psi+\hat{\psi}]] \\
& =\int \mathcal{D} \pi[\psi] \mathcal{D} \pi\left[\psi^{\prime}\right]\left\langle\ln \left[Z\left[\psi+\hat{\Psi}\left[\psi^{\prime}, \phi\right]\right]\right]\right\rangle_{\phi} \\
& =\int \mathcal{D} \pi[\psi] \mathcal{D} \pi\left[\psi^{\prime}\right]\left\langle\ln \left[Z_{2}\left[\psi, \psi^{\prime}, \phi\right]\right]\right\rangle_{\phi} .
\end{aligned}
$$

In the last step, the definition (26), (27) of $\left.\hat{\Psi}\left[\psi^{\prime}, \phi\right]\right)$ was invoked. We thus note that the first and second contribution to (71) can be combined so as to finally obtain (28).

The internal energy is obtained from the free energy via the relation $E(\beta)=\frac{\partial}{\partial \beta}(\beta f(\beta))$.

\section{Bethe-Peierls Method}

The replica-symmetric fixed point equations can be derived as resulting from a recursive evaluation of the partition function of the system described by an energy function of the form

$$
U_{\mathrm{int}}=\sum_{(i, j)} c_{i j} \phi_{i j}\left(u_{i}-u_{j}\right)+\sum_{i} V\left(u_{i}\right)
$$

assuming that the collection of nodes form a tree-like structure. Scalar degrees of freedom and site-independent on-site potentials $V$ are assumed for simplicity.

The restricted partition function computed at fixed value $u_{i}$ for the coordinate at $i$ is

$$
Z_{i}\left(u_{i}\right)=\exp \left\{-\beta V\left(u_{i}\right)\right\} \int \prod_{j \in \mathcal{N}(i)} \mathrm{d} u_{j} \exp \left\{-\beta \phi_{i j}\left(u_{i}-u_{j}\right)\right\} Z_{j \mid i}\left(u_{j}\right)
$$

in which $\mathcal{N}(i)$ denotes the set of vertices connected to vertex $i$, the $Z_{j \mid i}\left(u_{j}\right)$ are the restricted partition functions of the subtrees rooted in $j$ - exluding the tree rooted in node $i$ - evaluated 
at the value $u_{j}$ of the coordinate at the root-node $j$. If the system were tree-like, the $u_{j}$ integrals in this expression would factor,

$$
Z_{i}\left(u_{i}\right)=\exp \left\{-\beta V\left(u_{i}\right)\right\} \prod_{j \in \mathcal{N}(i)} \int \mathrm{d} u_{j} \exp \left\{-\beta \phi_{i j}\left(u_{i}-u_{j}\right)\right\} Z_{j \mid i}\left(u_{j}\right),
$$

and we would have the recursion

$$
Z_{j \mid i}\left(u_{j}\right)=\exp \left\{-\beta V\left(u_{j}\right)\right\} \prod_{k \in \mathcal{N}(j) \backslash i} \int \mathrm{d} u_{k} \exp \left\{-\beta \phi_{j k}\left(u_{j}-u_{k}\right)\right\} Z_{k \mid j}\left(u_{k}\right) .
$$

This recursion is the basis for the iterative Bethe-Peierls method for solving statistical mechanical problems on tree-like structures.

The system we are considering is, however, only locally tree-like, i.e. there are loops with lengths of $\mathcal{O}(\ln N)$. Factorization is, therfore, in principle only approximate. However, if the system has only a single thermodynamic state and correlations decay exponentially with distance (this is the situation of unbroken replica symmetry), then factorization would be asymptotically exact in the thermodynamic limit, as common ancestors of two sites neighbouring on a given site occur typically at distance $\mathcal{O}(\ln N)$ if the given site is removed, entailing that correlations are negligible in the thermodynamic limit.

One introduces cavity potentials $\hat{\psi}_{j \mid i}\left(u_{i}\right)$ associated with the factors appearing in (74) via

$$
\exp \left\{-\beta \hat{\psi}_{j \mid i}\left(u_{i}\right)\right\}=\int \mathrm{d} u_{j} \exp \left\{-\beta \phi_{i j}\left(u_{i}-u_{j}\right)\right\} Z_{j \mid i}\left(u_{j}\right)
$$

For the restricted partition functions $Z_{j \mid i}\left(u_{j}\right)$ of the sub-trees themselves we have

$$
Z_{j \mid i}\left(u_{j}\right)=\exp \left\{-\beta V\left(u_{j}\right)\right\} \prod_{k \in \mathcal{N}(j) \backslash i} \exp \left\{-\beta \hat{\psi}_{j \mid i}\left(u_{i}\right)\right\}
$$

In terms of the cavity potentials $\hat{\psi}_{j \mid i}$ and the free energies $\psi_{j \mid i}\left(u_{j}\right)$ associated with restricted sub-tree partition functions via

$$
Z_{j \mid i}\left(u_{j}\right)=\exp \left\{-\beta \psi_{j \mid i}\left(u_{j}\right)\right\}
$$

one has the recursion

$$
\begin{aligned}
\psi_{j \mid i}\left(u_{j}\right) & =V\left(u_{j}\right)+\sum_{k \in \mathcal{N}(j) \backslash i} \hat{\psi}_{k \mid j}\left(u_{j}\right) \\
\hat{\psi}_{k \mid j}\left(u_{j}\right) & =-\beta^{-1} \ln \int \mathrm{d} u_{k} \exp \left\{-\beta \phi_{j k}\left(u_{j}-u_{k}\right)-\beta \psi_{k \mid j}\left(u_{j}\right)\right\} \\
& \equiv-\beta^{-1} \ln Z_{\psi_{k \mid j} \phi_{j k}}
\end{aligned}
$$


The replica symmetric fixed-point equations derived in Sect. (3.1) are now recovered by observing that due to the locally varying coordination and the heterogeneity of the interaction potentials $\phi_{i j}$ associated with the edges $(i, j)$ of the tree-like graph, the cavity potentials and the restricted sub-tree free energies are random. Hence the iteration has to be formulated in a probabilistic setting as an iteration for probabilities of finding certain restricted sub-tree free energies $\psi$ and cavity potentials $\hat{\psi}$. For a homogeneously disordered (locally) tree-like graph this would lead to self-consistency conditions that have to hold in the thermodynamic limit. Using Eqs. (79) and (80) one finds

$$
\begin{aligned}
& \pi[\psi]=\sum_{L>0} \frac{L}{C} P_{C}(L) \int \prod_{k=1}^{L-1} \mathcal{D} \hat{\pi}\left[\hat{\psi}_{k}\right] \delta\left[\psi-\Psi_{L-1}\left[\left\{\hat{\psi}_{k}\right\}\right]\right] \\
& \hat{\pi}[\hat{\psi}]=\int \mathcal{D} \pi[\hat{\psi}]\langle\delta[\hat{\psi}-\hat{\Psi}[\psi, \phi]]\rangle_{\phi}
\end{aligned}
$$

for the self-consistency conditions, on a graph with connectivity distribution $P_{C}(L)$ of average coordination $C$, and $\Psi_{L-1}$ and $\hat{\Psi}\left[\psi, \phi_{\omega}\right]$ are as defined via (25) and (26).

\section{References}

[1] E. J. Donth. The Glass Transition: Relaxation Dynamics in Liquids and Disordered Materials. Springer Verlag, Berlin, 2001.

[2] W Götze. Aspects of structural glass transitions, Les Houches, Session LI, 3-28 juilliet 1989. In J. P. Hansen, D. Levesque, and J. Zinn-Justin, editors, Liquids, freezing and glass transition, pages 287-503, Amsterdam, 1991. North-Holland.

[3] M. D. Edinger, C. A. Angell, and S. R. Nagel. Supercooled liquids and glasses. J. Phys. Chem., 100:13200-13212, 1996.

[4] W. Kauzmann. The nature of the glassy state and the behavior of liquids at low temperatures. Chem. Rev., 43:219-256, 1948.

[5] J H Gibbs and E A DiMarzio. Nature of the glass transition and the glassy state. J. Chem. Phys., 28:373-383, 1958. Classic: Lattice theory for glass transition in polymers.

[6] G Adam and J H Gibbs. On the temperature dependence of cooperative relaxation properties in glass-forming liquids. J. Chem. Phys., 43:139-146, 1965.

[7] T. R. Kirkpatrick and P. G. Wolynes. Connections between some kinetic and equilibrium theories of the glass transition. Phys. Rev. A, 35:3072-3080, 1987. 
[8] T. R. Kirkpatrick and D. Thirumalai. Dynamics of the structural glass transition and the p-spin interaction spin-glass model. Phys. Rev. Lett., 58:2091-2094, 1987.

[9] T. R. Kirkpatrick and D. Thirumalai. p-spin-interaction spin-glass models: Connections with the structural glass problem. Phys. Rev. B, 36:5388-5397, 1987.

[10] A Crisanti and H J Sommers. The spherical $p$-spin interaction spin glass model: the statics. Z. Phys. B, 87:341-354, 1992.

[11] A Crisanti, H Horner, and H J Sommers. The spherical $p$-spin interaction spin-glass model - the dynamics. Z. Phys. B, 92:257-271, 1993.

[12] L F Cugliandolo and J Kurchan. Analytical solution of the off-equilibrium dynamics of a long- range spin-glass model. Phys. Rev. Lett., 71:173-176, 1993.

[13] J P Bouchaud and M Mézard. Self-induced quenched disorder - a model for the glasstransition. J. de Physique I (France), 4:1109-1114, 1994.

[14] E. Marinari, G. Parisi, and F. Ritort. Replica field theory for deterministic models: I. binary sequences with low autocorrelation. J. Phys. A, 27:7615-7645, 1994.

[15] E. Marinari, G. Parisi, and F. Ritort. Replica field theory for deterministic models. II. a non-random spin glass with glassy behaviour. J. Phys. A, 27:7647-7668, 1994.

[16] M. Mézard and G. Parisi. Thermodynamics of glasses: A first principles computation. Phys. Rev. Lett., 82:742-750, 1999.

[17] R. Monasson. Structural glass transition and the entropy of the metastable states. Phys. Rev. Lett., 75:2847-2850, 1995.

[18] S. Franz and G. Parisi. Phase diagram of coupled glassy systems: A mean-field study. Phys. Rev. Lett., 79:2486-2489, 1997.

[19] S. Franz and G. Parisi. Recipes for metastable states in spin glasses. J. Phys. I (France), 5:1401-1415, 1995.

[20] S. Franz, M. Mezard, F. Ricci-Tersenghi, M. Weigt, and R. Zecchina. A ferromagnet with a glass transition. Europhys. Lett., 55:465-471, 2001.

[21] G. Biroli and M. Mézard. Lattice glass models. Phys. Rev. Lett, 88:025501, 2002.

[22] A.K. Hartmann and M. Weigt. Glassy behavior induced by geometrical frustration in a hard-core lattice gas model. Europhys. Lett., 62:533-539, 2003.

[23] M. Pica Ciamarra, A. de Candia M. Tarzia, and A. Coniglio. A lattice glass model with no tendency to crystallize. Phys. Rev. E, 67:057105, 2003. 
[24] O. Rivoire, G. Biroli, O. Martin, and M. Mézard. Glass models on bethe lattices. Eur. Phys. J. B, 37:55-78, 2004.

[25] H. Hansen-Goos and M. Weigt. A hard-sphere model on generalized Bethe lattices: Statics. J. Stat. Mech., P04006, 2005.

[26] R. C. Zeller and R. O. Pohl. Thermal conductivity and specific heat of noncrystalline solids. Phys. Rev. B, 4:2029-2041, 1971.

[27] L. Piché, R. Maynard, S. Hunklinger, and J. Jäckle. Anomalous sound velocity in vitreous silica at very low temperatures. Phys. Rev. Lett., 32:1426-1429, 1974.

[28] P.W. Anderson, B. Halperin, and S. Varma. Anomalous low-temperature properties of glasses and spin glasses. Phil. Mag., 25:1-9, 1972.

[29] W.A. Phillips. Tunneling states in amorphous solids. J. Low Temp. Phys., 7:351-360, 1972.

[30] V. G. Karpov, M. I. Klinger, and F.N. Ignat'ev. Theory of the low-temperature anomalies in the thermal properties of amorphous structures. Sovj. Phys. JETP, 57:439-448, 1983.

[31] D. A. Parshin. Interactions of soft atomic potentials and universality of low-temperature properties of glasses. Phys. Rev. B, 49:9400-9418, 1994.

[32] M.A. Ramos and U. Buchenau. Soft-potential model. In P. Esquinazi, editor, Tunneling Systems in Amorphous and Crystalline Solids, page 527. Springer, Berlin, 1989.

[33] W. Schirmacher, G. Diezemann, and C. Ganter. Harmonic vibrational excitations in disordered solids and the Boson Peak. Phys. Rev. Lett., 81:136-139, 1998.

[34] T.S. Grigera, V. Martin-Mayor, G. Parisi, and P. Verrocchio. Phonon interpretation of the 'boson peak' in supercooled liquids. Nature, 422:289-292, 2003.

[35] C. C. Yu and A. J. Leggett. Low temperature properties of amorphous materials: Through a glass darkly. Comments Cond. Mat. Phys., 14:23-251, 1988.

[36] A. L. Burin and Yu. Kagan. Nature of the universal properties of amorphous solids. Sovj. Phys. JETP, 82:159-171, 1996.

[37] R. Kühn and U. Horstmann. Random matrix approach to glassy physics: Low temperatures and beyond. Phys. Rev. Lett., 78:4067-4070, 1997.

[38] R. Kühn and J. Urmann. Translational invariance in models for low-temperature properties of glasses. J. Phys. C, 12:6395-6403, 2000.

[39] R. Kühn. Universality in glassy low-temperature physics. Europhys. Lett., 62:313-319, 2003. 
[40] L.Viana and A.J. Bray. Phase diagrams for dilute spin glasses. J. Phys. C, 18:3037-3051, 1985 .

[41] R. Monasson. Optimization problems and replica symmetry breaking in finite connectivity spin-glasses. J. Phys. A, 31:513-529, 1998.

[42] M. Mézard and G. Parisi. The bethe lattice spin glass revisited. Eur. Phys. J. B, 20:217-233, 2001.

[43] A.K. Hartmann and M. Weigt. Phase transitions in combinatorial optimization problems. Wiley-VCH, Berlin, 2005.

[44] G. Semerjian and L. F. Cugliandolo. Dynamics of dilute disordered models: A solvable case. Europhys. Lett., 61:247-253, 2003.

[45] G. Semerjian and M. Weigt. Approximation schemes for the dynamics of diluted spin models: the ising ferromagnet on a bethe lattice. J. Phys. A, 37:5525-5546, 2004.

[46] H. Hansen-Goos and M. Weigt. A hard-sphere model on generalized Bethe lattices: Dynamics. J. Stat. Mech., P08001, 2005.

[47] J. P. L. Hatchett, I. Pérez Castillo, A. C. C. Coolen, and N. S. Skantzos. Dynamical replica analysis of disordered ising spin systems on finitely connected random graphs. Phys. Rev. Lett., 95:117204, 2005.

[48] G. Semerjian A. Montanari. From large scale rearrangements to mode coupling phenomenology. Phys. Rev. Lett., 94:247201, 2005.

[49] P.M. Goldbart, H.M. Castillo, and A. Zippelius. Randomly crosslinked macromolecular systems: Vulcanisation transition to and properties of the amorphous solid state. Adv. Phys., 45:393-468, 1996.

[50] A. Zippelius and P.M Goldbart. Vulcanized matter: A model glass? In A.P. Young, editor, Spin Glasses and Random Fields, pages 357-386. World Scientific, Singapore, 1998.

[51] K. Broderix, M. Weigt, and A. Zippelius. Towards finite dimensional gelation. Eur. Phys. J. B, 29:441-455, 2002.

[52] X. Mao, P. M. Goldbart, M. Mézard, and M. Weigt. Cavity approach to the random solid state. Phys. Rev. Lett., 95:148302, 1995.

[53] X. Mao, P. M. Goldbart, X. Xing, and A. Zippelius. Elastic heterogeneity of soft random solids. arXiv:cond-mat/0610407. 
[54] K. Y. M. Wong and D. Sherrington. Graph bipartitioning and spin glasses on a random network of fixed finite valence. J. Phys. A, 20:L793-L799, 1987.

[55] J. Jäckle. On the ultrasound attenuation in glasses at low temperatures. Z. Phys., 257:212$223,1972$. 